\title{
In-source CID ramping (InCIDR) and Co-variant ion analysis of hydrophilic interaction chromatography (HILIC) metabolomics
}

Xiaoyang Su, ${ }^{*}, 1,2$ Eric Chiles, ${ }^{2}$ Sara Maimouni, ${ }^{2,3}$ Fredric E. Wondisford, ${ }^{1}$ Wei-Xing Zong, ${ }^{2,3}$ Chi Song ${ }^{*}, 4$

1. Department of Medicine, Rutgers-Robert Wood Johnson Medical School, New Brunswick, NJ, 08901, USA

2. Rutgers Cancer Institute of New Jersey, New Brunswick, NJ 08903, USA

3. Department of Chemical Biology, Ernest Mario School of Pharmacy, Rutgers University, Piscataway, New Jersey 08854, USA.

4. Division of Biostatistics, College of Public Health, The Ohio State University, Columbus, OH 43210, USA

\section{SUPPORT INFORMATION}

Figure S1. The grouping performance of the adduct annotation tools

Figure S2. Statistics on adduct annotation

Figure S3. Rate of correct prediction of metabolite neutral mass

Table S1. The complete annotation of co-variant ions of 10 metabolites in the standard mixture

Table S2. The rank correlation coefficients and the ranks scores for Glc6P and NAD calculated by InCIDR

File S1. The extended adduct list for CliqueMS (Negative ionization mode)

File S2. The extended adduct list for CliqueMS (Positive ionization mode) 


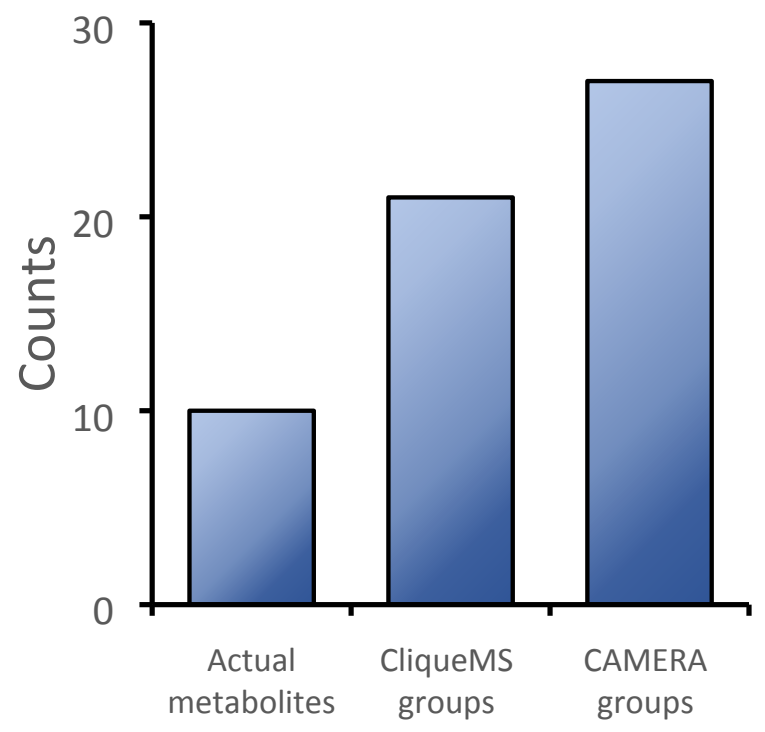

Figure S1. The grouping performance of the adduct annotation tools. We used the dataset from the standard mixture to test the grouping algorithms in these tools. The standard mixture has 10 metabolites. CliqueMS put the resulting adduct ions into 20 groups, while CAMERA put the resulting adduct ions into 27 groups. 
Annotated adduct ions

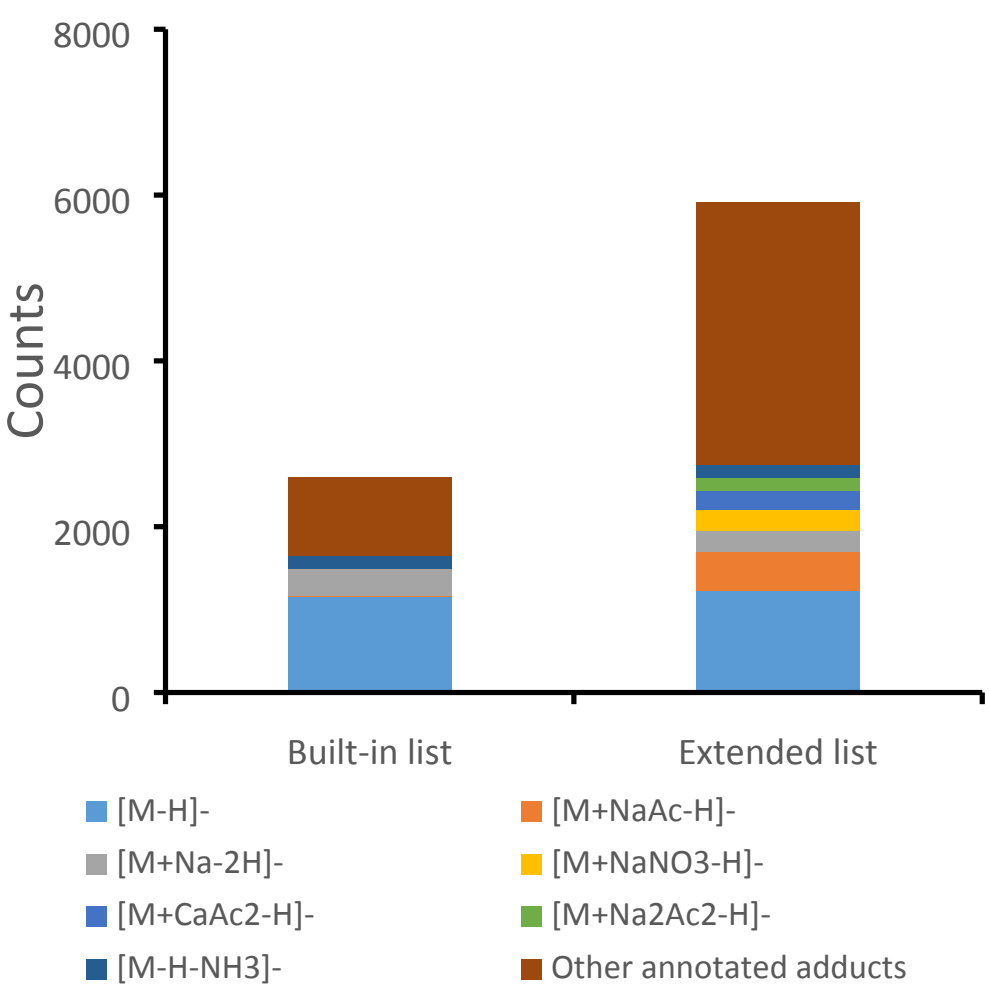

Figure S2. Statistics on adduct annotation. The bars show the number of annotated adduct ions in the L3.6 cell lysate dataset using CliqueMS with both the built-in adduct list and the extended adduct list. The total feature number in the dataset is 11,055 . 


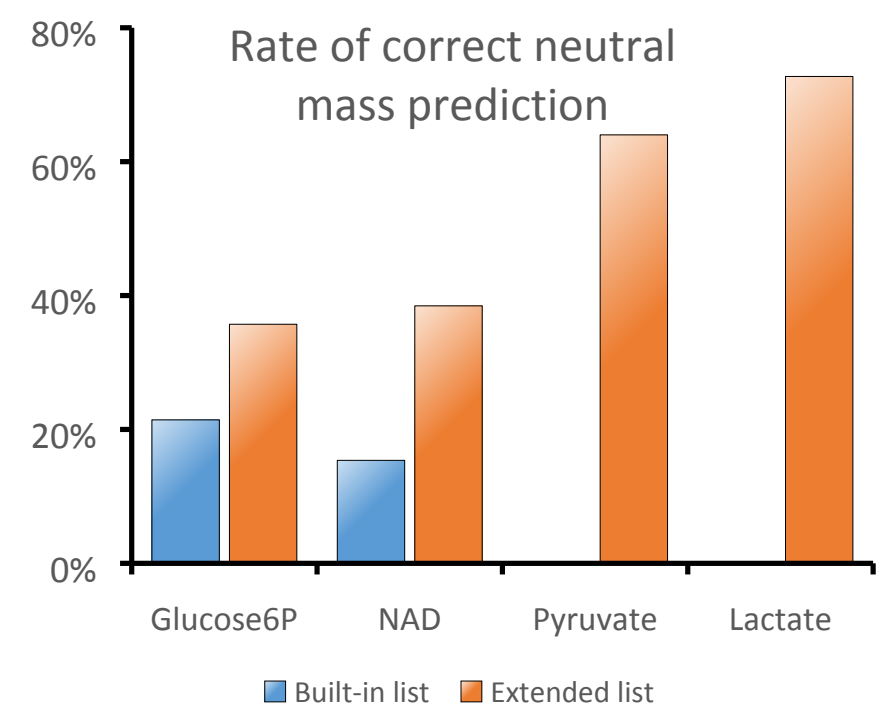

Figure S3. Rate of prediction of metabolite neutral mass. The bars compare the rate of correct neutral mass prediction by CliqueMS using both the built-in adduct list and the extended adduct list for the key metabolites. 
Table S1. The complete annotation of co-variant ions of 10 metabolites in the standard mixture. * These adduct ions do not have their counterparts detected in ${ }^{2} \mathrm{H}_{3}$-Ac mobile phase. They are annotated as $\mathrm{Mg}^{2+}$ containing adduct ions. Presumably, the ${ }^{2} \mathrm{H}_{3}-\mathrm{Ac}^{2}$ has much lower level of $\mathrm{Mg}^{2+}$ so that these adduct ions were not formed.

\begin{tabular}{|c|c|c|c|c|c|c|c|c|}
\hline \multicolumn{9}{|c|}{ Pyruvate } \\
\hline $\begin{array}{c}\text { Observed } \\
\mathrm{m} / \mathrm{z}\end{array}$ & $\begin{array}{c}\text { Relative } \\
\text { Abundance }\end{array}$ & $\Delta \mathrm{m}$ & Chemical annotation & $\begin{array}{c}\text { Calculated } \\
\mathrm{m} / \mathrm{z}\end{array}$ & $\begin{array}{c}\text { Mass } \\
\text { accuracy } \\
\text { (ppm) }\end{array}$ & $\begin{array}{c}\text { Observed } \\
\mathrm{m} / \mathrm{z} \text { in } \\
{ }^{2} \mathrm{H}_{3}-\mathrm{Ac}\end{array}$ & $\begin{array}{c}\text { Calculated } \\
\mathrm{m} / \mathrm{z} \text { in } \\
{ }^{2} \mathrm{H}_{3}-\mathrm{Ac} \\
\end{array}$ & $\begin{array}{c}\text { Mass } \\
\text { accuracy in } \\
{ }^{2} \mathrm{H}_{3}-\mathrm{Ac}(\mathrm{ppm})\end{array}$ \\
\hline 86.0248 & $0.86 \%$ & -0.9840 & {$\left[\mathrm{M}-\mathrm{H}_{2} \mathrm{O}+\mathrm{NH}_{3}-\mathrm{H}\right]^{-}$} & 86.0248 & 0.5 & 86.0247 & 86.0248 & -0.6 \\
\hline 87.0087 & $100.00 \%$ & 0.0000 & {$[\mathrm{M}-\mathrm{H}]^{-}$} & 87.0088 & -0.3 & 87.0087 & 87.0088 & -1.4 \\
\hline 88.0122 & $3.21 \%$ & 1.0034 & ${ }^{13} \mathrm{C}_{1}-[\mathrm{M}-\mathrm{H}]^{-}$ & 88.0121 & 0.7 & 88.0120 & 88.0121 & -1.4 \\
\hline 169.0118 & $30.15 \%$ & 82.0030 & {$[\mathrm{M}+\mathrm{NaAc}-\mathrm{H}]^{-}$} & 169.0118 & -0.5 & 172.0307 & 172.0307 & 0.2 \\
\hline 170.0149 & $1.40 \%$ & 83.0062 & $\left.{ }^{13} \mathrm{C}_{1}-[\mathrm{M}+\mathrm{NaAc}-\mathrm{H}]\right]^{-}$ & 170.0152 & -1.6 & 173.0340 & 173.0340 & -0.2 \\
\hline 171.9863 & $92.93 \%$ & 84.9776 & {$\left[\mathrm{M}+\mathrm{NaNO}_{3}-\mathrm{H}\right]^{-}$} & 171.9864 & -0.1 & 171.9864 & 171.9864 & 0.3 \\
\hline 172.9899 & $3.19 \%$ & 85.9812 & ${ }^{13} \mathrm{C}_{1}-\left[\mathrm{M}+\mathrm{NaNO}_{3}-\mathrm{H}\right]^{-}$ & 172.9897 & 1.2 & 172.9896 & 172.9897 & -0.6 \\
\hline 197.0068 & $13.22 \%$ & 109.9981 & {$[2 \mathrm{M}+\mathrm{Na}-2 \mathrm{H}]^{-}$} & 197.0068 & 0.1 & 197.0067 & 197.0068 & -0.5 \\
\hline 198.0102 & $0.79 \%$ & 111.0015 & ${ }^{13} \mathrm{C}_{1}-[2 \mathrm{M}+\mathrm{Na}-2 \mathrm{H}]-$ & 198.0101 & 0.5 & 198.0101 & 198.0101 & 0.0 \\
\hline 229.0204 & $0.93 \%$ & 142.0116 & {$\left[\mathrm{M}+\mathrm{MgAc} \mathrm{c}_{2}-\mathrm{H}\right]^{-}$} & 229.0204 & -0.3 & $N^{*}$ & 235.0581 & $N^{*}$ \\
\hline 244.9977 & $8.68 \%$ & 157.9890 & {$\left[\mathrm{M}+\mathrm{CaAc} \mathrm{C}_{2}-\mathrm{H}\right]^{-}$} & 244.9980 & -1.1 & 251.0356 & 251.0356 & -0.1 \\
\hline 251.0145 & $2.86 \%$ & 164.0058 & {$\left[\mathrm{M}+\mathrm{Na}_{2} \mathrm{Ac}_{2}-\mathrm{H}\right]^{-}$} & 251.0149 & -1.5 & 257.0526 & 257.0526 & 0.1 \\
\hline 260.9737 & $5.85 \%$ & 173.9650 & {$\left[\mathrm{M}+\mathrm{H}_{6} \mathrm{Si}_{2} \mathrm{O}_{7}-\mathrm{H}\right]^{-}$} & 260.9740 & -1.0 & 260.9738 & 260.9740 & -0.7 \\
\hline 261.9739 & $0.53 \%$ & 174.9651 & ${ }^{29} \mathrm{Si}_{1}-\left[\mathrm{M}+\mathrm{H}_{6} \mathrm{Si}_{2} \mathrm{O}_{7}-\mathrm{H}\right]^{-}$ & 261.9735 & 1.2 & 261.9727 & 261.9735 & -3.2 \\
\hline 327.0005 & $4.88 \%$ & 239.9918 & {$\left[\mathrm{M}+\mathrm{NaCaAc} c_{3}-\mathrm{H}\right]^{-}$} & 327.0010 & -1.6 & 336.0575 & 336.0575 & -0.1 \\
\hline 333.0176 & $2.40 \%$ & 246.0089 & {$\left[\mathrm{M}+\mathrm{Na} 3 \mathrm{Ac}_{3}-\mathrm{H}\right]^{-}$} & 333.0180 & -1.1 & 342.0743 & 342.0745 & -0.5 \\
\hline 338.9517 & $0.84 \%$ & 251.9430 & {$\left[\mathrm{M}+\mathrm{H}_{8} \mathrm{Si}_{3} \mathrm{O}_{10}-\mathrm{H}\right]^{-}$} & 338.9513 & 1.2 & 338.9512 & 338.9513 & -0.3 \\
\hline 409.0039 & $3.20 \%$ & 321.9952 & {$\left[\mathrm{M}+\mathrm{Na}_{2} \mathrm{CaAc}_{4}-\mathrm{H}\right]^{-}$} & 409.0041 & -0.5 & 421.0795 & 421.0794 & 0.1 \\
\hline 415.0199 & $1.25 \%$ & 328.0111 & {$\left[\mathrm{M}+\mathrm{Na}_{4} \mathrm{Ac}_{4}-\mathrm{H}\right]^{-}$} & 415.0211 & -2.9 & 427.0964 & 427.0964 & 0.0 \\
\hline 491.0062 & $2.16 \%$ & 403.9975 & {$\left[\mathrm{M}+\mathrm{Na}_{3} \mathrm{CaAC}_{5}-\mathrm{H}\right]^{-}$} & 491.0072 & -2.0 & 506.1014 & 506.1013 & 0.1 \\
\hline 566.9928 & $0.87 \%$ & 479.9841 & {$\left[\mathrm{M}+\mathrm{Na}_{2} \mathrm{Ca}_{2} \mathrm{Ac}_{6}-\mathrm{H}\right]^{-}$} & 566.9933 & -0.9 & 585.1055 & 585.1063 & -1.4 \\
\hline 573.0108 & $1.04 \%$ & 486.0021 & {$\left[\mathrm{M}+\mathrm{Na}_{4} \mathrm{CaAc} \mathrm{C}_{6}-\mathrm{H}\right]^{-}$} & 573.0103 & 0.9 & 591.1229 & 591.1232 & -0.6 \\
\hline
\end{tabular}




\begin{tabular}{|c|c|c|c|c|c|c|c|c|}
\hline \multicolumn{9}{|c|}{ Lactate } \\
\hline $\begin{array}{c}\text { Observed } \\
\mathrm{m} / \mathrm{z}\end{array}$ & $\begin{array}{c}\text { Relative } \\
\text { Abundance }\end{array}$ & $\Delta \mathrm{m}$ & Chemical annotation & $\begin{array}{c}\text { Calculated } \\
\mathrm{m} / \mathrm{z}\end{array}$ & $\begin{array}{c}\text { Mass } \\
\text { accuracy } \\
\text { (ppm) }\end{array}$ & $\begin{array}{c}\text { Observed } \\
\mathrm{m} / \mathrm{z} \text { in } \\
{ }^{2} \mathrm{H}_{3}-\mathrm{Ac}\end{array}$ & $\begin{array}{c}\text { Calculated } \\
\mathrm{m} / \mathrm{z} \text { in } \\
{ }^{2} \mathrm{H}_{3}-\mathrm{Ac}\end{array}$ & $\begin{array}{c}\text { Mass } \\
\text { accuracy in } \\
{ }^{2} \mathrm{H}_{3}-\mathrm{Ac}(\mathrm{ppm})\end{array}$ \\
\hline 89.0244 & $100.00 \%$ & 0.0000 & {$[\mathrm{M}-\mathrm{H}]^{-}$} & 89.0244 & -0.7 & 89.0245 & 89.0244 & 0.7 \\
\hline 90.0276 & $3.32 \%$ & 1.0032 & ${ }^{13} \mathrm{C}_{1}-[\mathrm{M}-\mathrm{H}]^{-}$ & 90.0278 & -2.2 & 90.0278 & 90.0278 & 0.0 \\
\hline 91.0289 & $0.69 \%$ & 2.0045 & ${ }^{18} \mathrm{O}_{1}-[\mathrm{M}-\mathrm{H}]^{-}$ & 91.0287 & 2.5 & 91.0287 & 91.0287 & 0.1 \\
\hline 148.9905 & $0.40 \%$ & 59.9661 & {$\left[\mathrm{M}+\mathrm{SiO}_{2}-\mathrm{H}\right]^{-}$} & 148.9912 & -4.8 & 148.9910 & 148.9912 & -1.1 \\
\hline 171.0276 & $13.20 \%$ & 82.0032 & {$[\mathrm{M}+\mathrm{NaAc}-\mathrm{H}]^{-}$} & 171.0275 & 0.6 & 174.0462 & 174.0463 & -0.7 \\
\hline 172.0306 & $0.56 \%$ & 83.0062 & $\left.{ }^{13} \mathrm{C}_{1}-[\mathrm{M}+\mathrm{NaAC}-\mathrm{H}]\right]^{-}$ & 172.0309 & -1.7 & 175.0496 & 175.0497 & -0.5 \\
\hline 174.0020 & $36.30 \%$ & 84.9776 & {$\left[\mathrm{M}+\mathrm{NaNO}_{3}-\mathrm{H}\right]^{-}$} & 174.0020 & 0.0 & 174.0019 & 174.0020 & -0.6 \\
\hline 175.0061 & $1.00 \%$ & 85.9817 & ${ }^{13} \mathrm{C}_{1}-\left[\mathrm{M}+\mathrm{NaNO}_{3}-\mathrm{H}\right]^{-}$ & 175.0054 & 4.0 & 175.0052 & 175.0054 & -0.9 \\
\hline 177.9570 & $0.44 \%$ & 88.9327 & {$[\mathrm{M}+\mathrm{FeO}(\mathrm{OH})-\mathrm{H}]^{-}$} & 177.9570 & 0.1 & 177.9572 & 177.9570 & 1.1 \\
\hline 185.0120 & $0.87 \%$ & 95.9876 & {$\left[\mathrm{M}+\mathrm{H}_{4} \mathrm{SiO}_{4}-\mathrm{H}\right]^{-}$} & 185.0123 & -1.7 & 185.0123 & 185.0123 & 0.0 \\
\hline 201.0387 & $6.43 \%$ & 112.0143 & {$[2 \mathrm{M}+\mathrm{Na}-2 \mathrm{H}]^{-}$} & 201.0381 & 3.1 & 201.0381 & 201.0381 & 0.2 \\
\hline 202.0426 & $0.43 \%$ & 113.0183 & ${ }^{13} \mathrm{C}_{1}-[2 \mathrm{M}+\mathrm{Na}-2 \mathrm{H}]^{-}$ & 202.0414 & 6.0 & 202.0415 & 202.0414 & 0.4 \\
\hline 231.0369 & $0.92 \%$ & 142.0126 & {$\left[\mathrm{M}+\mathrm{MgAc} \mathrm{c}_{2}-\mathrm{H}\right]^{-}$} & 231.0361 & 3.7 & $N^{*}$ & 231.0361 & $N^{*}$ \\
\hline 247.0135 & $5.70 \%$ & 157.9892 & {$\left[\mathrm{M}+\mathrm{CaAc}_{2}-\mathrm{H}\right]^{-}$} & 247.0136 & -0.4 & 253.0513 & 253.0513 & 0.1 \\
\hline 253.0312 & $1.09 \%$ & 164.0069 & {$\left[\mathrm{M}+\mathrm{Na}_{2} \mathrm{Ac}_{2}-\mathrm{H}\right]^{-}$} & 253.0306 & 2.6 & 259.0681 & 259.0682 & -0.5 \\
\hline 262.9891 & $3.02 \%$ & 173.9648 & {$\left[\mathrm{M}+\mathrm{H}_{6} \mathrm{Si}_{2} \mathrm{O}_{7}-\mathrm{H}\right]^{-}$} & 262.9896 & -1.8 & 262.9896 & 262.9896 & -0.1 \\
\hline 313.0396 & $0.51 \%$ & 224.0152 & {$\left[\mathrm{M}+\mathrm{NaMgAc}_{3}-\mathrm{H}\right]^{-}$} & 313.0391 & 1.3 & ND* & 322.0956 & $\mathrm{ND}^{*}$ \\
\hline 329.0170 & $2.78 \%$ & 239.9926 & {$\left[\mathrm{M}+\mathrm{NaCaAc}{ }_{3}-\mathrm{H}\right]^{-}$} & 329.0167 & 0.9 & 338.0732 & 338.0732 & 0.1 \\
\hline 411.0196 & $1.37 \%$ & 321.9953 & {$\left[\mathrm{M}+\mathrm{Na}_{2} \mathrm{CaAc}_{4}-\mathrm{H}\right]^{-}$} & 411.0198 & -0.4 & 423.0950 & 423.0951 & -0.2 \\
\hline 493.0235 & $0.71 \%$ & 403.9991 & {$\left[\mathrm{M}+\mathrm{Na}_{3} \mathrm{CaAc}_{5}-\mathrm{H}\right]^{-}$} & 493.0228 & 1.3 & 508.1173 & 508.1170 & 0.6 \\
\hline
\end{tabular}




\begin{tabular}{|c|c|c|c|c|c|c|c|c|}
\hline \multicolumn{9}{|c|}{ Leucine } \\
\hline $\begin{array}{c}\text { Observed } \\
\mathrm{m} / \mathrm{z}\end{array}$ & $\begin{array}{c}\text { Relative } \\
\text { Abundance }\end{array}$ & $\Delta \mathrm{m}$ & Chemical annotation & $\begin{array}{c}\text { Calculated } \\
\mathrm{m} / \mathrm{z}\end{array}$ & $\begin{array}{c}\text { Mass } \\
\text { accuracy } \\
(\mathrm{ppm})\end{array}$ & $\begin{array}{c}\text { Observed } \\
\mathrm{m} / \mathrm{z} \text { in } \\
{ }^{2} \mathrm{H}_{3}-\mathrm{Ac}\end{array}$ & $\begin{array}{c}\text { Calculated } \\
\mathrm{m} / \mathrm{z} \text { in } \\
{ }^{2} \mathrm{H}_{3}-\mathrm{Ac}\end{array}$ & $\begin{array}{c}\text { Mass } \\
\text { accuracy in } \\
{ }^{2} \mathrm{H}_{3}-\mathrm{Ac}(\mathrm{ppm})\end{array}$ \\
\hline 130.0873 & $100.00 \%$ & 0.0000 & {$[\mathrm{M}-\mathrm{H}]^{-}$} & 130.0874 & -0.4 & 130.0873 & 130.0874 & -0.8 \\
\hline 131.0907 & $6.63 \%$ & 1.0034 & ${ }^{13} \mathrm{C}_{1}-[\mathrm{M}-\mathrm{H}]^{-}$ & 131.0907 & -0.1 & 131.0906 & 131.0907 & -0.8 \\
\hline 132.0918 & $0.44 \%$ & 2.0045 & $\left.{ }^{18} \mathrm{O}_{1}-[\mathrm{M}-\mathrm{H}]\right]^{-}$ & 132.0916 & 1.5 & 132.0914 & 132.0916 & -1.5 \\
\hline 170.0800 & $0.21 \%$ & 39.9927 & {$[\mathrm{M}+\mathrm{NaOH}-\mathrm{H}]^{-}$} & 170.0796 & 2.6 & 170.0798 & 170.0796 & 1.4 \\
\hline 212.0905 & $11.64 \%$ & 82.0032 & {$[\mathrm{M}+\mathrm{NaAc}-\mathrm{H}]^{-}$} & 212.0904 & 0.3 & 215.1092 & 215.1093 & -0.3 \\
\hline 213.0935 & $1.01 \%$ & 83.0062 & ${ }^{13} \mathrm{C}_{1}-[\mathrm{M}+\mathrm{NaAc}-\mathrm{H}]^{-}$ & 213.0938 & -1.3 & 216.1124 & 216.1126 & -1.0 \\
\hline 215.0649 & $3.12 \%$ & 84.9776 & {$\left[\mathrm{M}+\mathrm{NaNO}_{3}-\mathrm{H}\right]^{-}$} & 215.0649 & -0.2 & 215.0649 & 215.0649 & -0.2 \\
\hline 226.0753 & $0.92 \%$ & 95.9880 & {$\left[\mathrm{M}+\mathrm{H}_{4} \mathrm{SiO}_{4}-\mathrm{H}\right]^{-}$} & 226.0752 & 0.3 & 226.0745 & 226.0752 & -3.3 \\
\hline 228.0650 & $0.21 \%$ & 97.9777 & {$[\mathrm{M}+\mathrm{KAc}-\mathrm{H}]^{-}$} & 228.0644 & 2.8 & 231.0831 & 231.0832 & -0.4 \\
\hline 272.0983 & $0.35 \%$ & 142.0110 & {$\left[\mathrm{M}+\mathrm{MgAc}_{2}-\mathrm{H}\right]^{-}$} & 272.0990 & -2.6 & $\mathrm{ND}^{*}$ & 278.1367 & $N^{*}$ \\
\hline 283.1639 & $0.69 \%$ & 153.0766 & {$[2 \mathrm{M}+\mathrm{Na}-2 \mathrm{H}]^{-}$} & 283.1639 & -0.1 & 283.1638 & 283.1639 & -0.5 \\
\hline 288.0765 & $5.52 \%$ & 157.9892 & {$\left[\mathrm{M}+\mathrm{CaAc} \mathrm{C}_{2}-\mathrm{H}\right]^{-}$} & 288.0766 & -0.2 & 294.1142 & 294.1142 & 0.0 \\
\hline 289.0794 & $0.57 \%$ & 158.9921 & ${ }^{13} \mathrm{C}_{1}-\left[\mathrm{M}+\mathrm{CaAc} \mathrm{C}_{2}-\mathrm{H}\right]-$ & 289.0799 & -1.8 & 295.1174 & 295.1176 & -0.6 \\
\hline 294.0934 & $1.60 \%$ & 164.0061 & {$\left[\mathrm{M}+\mathrm{Na}_{2} \mathrm{Ac}_{2}-\mathrm{H}\right]^{-}$} & 294.0935 & -0.3 & 300.1311 & 300.1312 & -0.2 \\
\hline 304.0530 & $0.59 \%$ & 173.9657 & {$\left[\mathrm{M}+\mathrm{H}_{6} \mathrm{Si}_{2} \mathrm{O}_{7}-\mathrm{H}\right]^{-}$} & 304.0526 & 1.4 & 304.0525 & 304.0526 & -0.2 \\
\hline 359.1497 & $0.26 \%$ & 229.0624 & {$[2 \mathrm{M}+\mathrm{CaAc}-2 \mathrm{H}]^{-}$} & 359.1501 & -1.0 & 362.1686 & 362.1689 & -0.8 \\
\hline 370.0794 & $2.65 \%$ & 239.9921 & {$\left[\mathrm{M}+\mathrm{NaCaAc} c_{3}-\mathrm{H}\right]^{-}$} & 370.0796 & -0.6 & 379.1360 & 379.1361 & -0.3 \\
\hline 371.0840 & $0.31 \%$ & 240.9967 & ${ }^{13} \mathrm{C}_{1}-\left[\mathrm{M}+\mathrm{NaCaAC} \mathrm{C}_{3}-\mathrm{H}\right]^{-}$ & 371.0830 & 2.7 & 380.1393 & 380.1395 & -0.4 \\
\hline 376.0964 & $0.66 \%$ & 246.0091 & {$\left[\mathrm{M}+\mathrm{Na}_{3} \mathrm{Ac}_{3}-\mathrm{H}\right]^{-}$} & 376.0966 & -0.5 & 385.1531 & 385.1531 & 0.1 \\
\hline 441.1535 & $0.24 \%$ & 311.0662 & {$\left[2 \mathrm{M}+\mathrm{NaCaAc} c_{2}-2 \mathrm{H}\right]^{-}$} & 441.1531 & 0.9 & 447.1908 & 447.1908 & 0.0 \\
\hline 446.0658 & $0.67 \%$ & 315.9785 & {$\left[\mathrm{M}+\mathrm{Ca}_{2} \mathrm{Ac}_{4}-\mathrm{H}\right]^{-}$} & 446.0658 & 0.1 & 458.1413 & 458.1411 & 0.5 \\
\hline 452.0826 & $1.22 \%$ & 321.9953 & {$\left[\mathrm{M}+\mathrm{Na}_{2} \mathrm{CaAC}_{4}-\mathrm{H}\right]^{-}$} & 452.0827 & -0.2 & 464.1583 & 464.1580 & 0.6 \\
\hline 458.1010 & $0.26 \%$ & 328.0137 & {$\left[\mathrm{M}+\mathrm{Na}_{4} \mathrm{Ac}_{4}-\mathrm{H}\right]^{-}$} & 458.0997 & 2.9 & 470.1749 & 470.1750 & -0.1 \\
\hline 523.1558 & $0.16 \%$ & 393.0685 & {$\left[2 \mathrm{M}+\mathrm{Na}_{2} \mathrm{CaAc}_{3}-2 \mathrm{H}\right]^{-}$} & 523.1562 & -0.8 & 532.2123 & 532.2127 & -0.7 \\
\hline 528.0689 & $0.63 \%$ & 397.9816 & {$\left[\mathrm{M}+\mathrm{NaCa}_{2} \mathrm{Ac}_{5}-\mathrm{H}\right]^{-}$} & 528.0688 & 0.1 & 543.1633 & 543.1630 & 0.6 \\
\hline 534.0880 & $0.61 \%$ & 404.0007 & {$\left[\mathrm{M}+\mathrm{Na}_{3} \mathrm{CaAc}_{5}-\mathrm{H}\right]^{-}$} & 534.0858 & 4.2 & 549.1798 & 549.1799 & -0.2 \\
\hline 610.0726 & $0.39 \%$ & 479.9853 & {$\left[\mathrm{M}+\mathrm{Na}_{2} \mathrm{Ca}_{2} \mathrm{Ac}_{6}-\mathrm{H}\right]^{-}$} & 610.0719 & 1.1 & 628.1842 & 628.1849 & -1.1 \\
\hline
\end{tabular}




\begin{tabular}{|c|c|c|c|c|c|c|c|c|}
\hline \multicolumn{9}{|c|}{ Isoleucine } \\
\hline $\begin{array}{c}\text { Observed } \\
\mathrm{m} / \mathrm{z}\end{array}$ & $\begin{array}{c}\text { Relative } \\
\text { Abundance }\end{array}$ & $\Delta \mathrm{m}$ & Chemical annotation & $\begin{array}{c}\text { Calculated } \\
\mathrm{m} / \mathrm{z}\end{array}$ & $\begin{array}{c}\text { Mass } \\
\text { accuracy } \\
\text { (ppm) }\end{array}$ & $\begin{array}{c}\text { Observed } \\
\mathrm{m} / \mathrm{z} \text { in } \\
{ }^{2} \mathrm{H}_{3}-\mathrm{Ac}\end{array}$ & $\begin{array}{c}\text { Calculated } \\
\mathrm{m} / \mathrm{z} \text { in } \\
{ }^{2} \mathrm{H}_{3}-\mathrm{Ac} \\
\end{array}$ & $\begin{array}{c}\text { Mass } \\
\text { accuracy in } \\
{ }^{2} \mathrm{H}_{3}-\mathrm{Ac}(\mathrm{ppm})\end{array}$ \\
\hline 130.0872 & $100.00 \%$ & 0.0000 & {$[\mathrm{M}-\mathrm{H}]^{-}$} & 130.0874 & -1.2 & 130.0873 & 130.0874 & -0.8 \\
\hline 131.0908 & $6.61 \%$ & 1.0036 & ${ }^{13} \mathrm{C}_{1}-[\mathrm{M}-\mathrm{H}]^{-}$ & 131.0907 & 0.7 & 131.0906 & 131.0907 & -0.8 \\
\hline 132.0921 & $0.41 \%$ & 2.0049 & ${ }^{18} \mathrm{O}_{1-}[\mathrm{M}-\mathrm{H}]^{-}$ & 132.0916 & 3.8 & 132.0914 & 132.0916 & -1.5 \\
\hline 170.0812 & $0.18 \%$ & 39.9940 & {$[\mathrm{M}+\mathrm{NaOH}-\mathrm{H}]^{-}$} & 170.0796 & 9.6 & 170.0798 & 170.0796 & 1.4 \\
\hline 212.0905 & $11.69 \%$ & 82.0033 & {$[\mathrm{M}+\mathrm{NaAc}-\mathrm{H}]^{-}$} & 212.0904 & 0.3 & 215.1093 & 215.1093 & 0.2 \\
\hline 213.0938 & $0.98 \%$ & 83.0066 & ${ }^{13} \mathrm{C}_{1}-[\mathrm{M}+\mathrm{NaAC}-\mathrm{H}]$ & 213.0938 & 0.1 & 216.1125 & 216.1126 & -0.5 \\
\hline 226.0747 & $0.93 \%$ & 95.9875 & {$\left[\mathrm{M}+\mathrm{H}_{4} \mathrm{SiO}_{4}-\mathrm{H}\right]^{-}$} & 226.0752 & -2.4 & 226.0745 & 226.0752 & -3.3 \\
\hline 272.0992 & $0.29 \%$ & 142.0120 & {$\left[\mathrm{M}+\mathrm{MgAc}_{2}-\mathrm{H}\right]^{-}$} & 272.0990 & 0.7 & ND* & 278.1367 & ND* \\
\hline 283.1641 & $0.59 \%$ & 153.0769 & {$[2 \mathrm{M}+\mathrm{Na}-2 \mathrm{H}]^{-}$} & 283.1639 & 0.6 & 283.1640 & 283.1639 & 0.2 \\
\hline 288.0765 & $5.91 \%$ & 157.9893 & {$\left[\mathrm{M}+\mathrm{CaAc} \mathrm{C}_{2}-\mathrm{H}\right]^{-}$} & 288.0766 & -0.2 & 294.1143 & 294.1142 & 0.3 \\
\hline 289.0798 & $0.60 \%$ & 158.9926 & ${ }^{13} \mathrm{C}_{1}-\left[\mathrm{M}+\mathrm{CaAc} \mathrm{C}_{2}-\mathrm{H}\right]^{-}$ & 289.0799 & -0.4 & 295.1174 & 295.1176 & -0.6 \\
\hline 294.0931 & $1.62 \%$ & 164.0059 & {$\left[\mathrm{M}+\mathrm{Na}_{2} \mathrm{Ac}_{2}-\mathrm{H}\right]^{-}$} & 294.0935 & -1.4 & 300.1313 & 300.1312 & 0.5 \\
\hline 304.0525 & $0.55 \%$ & 173.9653 & {$\left[\mathrm{M}+\mathrm{H}_{6} \mathrm{Si}_{2} \mathrm{O}_{7}-\mathrm{H}\right]^{-}$} & 304.0526 & -0.2 & 304.0527 & 304.0526 & 0.5 \\
\hline 359.1507 & $0.22 \%$ & 229.0635 & {$[2 \mathrm{M}+\mathrm{CaAc}-2 \mathrm{H}]^{-}$} & 359.1501 & 1.8 & 362.1690 & 362.1689 & 0.3 \\
\hline 370.0795 & $2.75 \%$ & 239.9923 & {$\left[\mathrm{M}+\mathrm{NaCaAc} c_{3}-\mathrm{H}\right]^{-}$} & 370.0796 & -0.4 & 379.1363 & 379.1361 & 0.5 \\
\hline 371.0815 & $0.30 \%$ & 240.9943 & ${ }^{13} \mathrm{C}_{1}-\left[\mathrm{M}+\mathrm{NaCaAc} \mathrm{C}_{3}-\mathrm{H}\right]^{-}$ & 371.0830 & -4.0 & 380.1399 & 380.1395 & 1.1 \\
\hline 376.0951 & $0.63 \%$ & 246.0079 & {$\left[\mathrm{M}+\mathrm{Na}_{3} \mathrm{Ac}_{3}-\mathrm{H}\right]^{-}$} & 376.0966 & -3.9 & 385.1532 & 385.1531 & 0.4 \\
\hline 446.0656 & $0.78 \%$ & 315.9784 & {$\left[\mathrm{M}+\mathrm{Ca}_{2} \mathrm{Ac}_{4}-\mathrm{H}\right]^{-}$} & 446.0658 & -0.3 & 458.1415 & 458.1411 & 0.9 \\
\hline 452.0825 & $1.33 \%$ & 321.9953 & {$\left[\mathrm{M}+\mathrm{Na}_{2} \mathrm{CaAc}_{4}-\mathrm{H}\right]^{-}$} & 452.0827 & -0.4 & 464.1582 & 464.1580 & 0.4 \\
\hline 528.0654 & $0.73 \%$ & 397.9782 & {$\left[\mathrm{M}+\mathrm{NaCa}_{2} \mathrm{Ac}_{5}-\mathrm{H}\right]^{-}$} & 528.0688 & -6.5 & 543.1630 & 543.1630 & 0.0 \\
\hline 534.0847 & $0.66 \%$ & 403.9975 & {$\left[\mathrm{M}+\mathrm{Na}_{3} \mathrm{CaAc}_{5}-\mathrm{H}\right]^{-}$} & 534.0858 & -2.0 & 549.1801 & 549.1799 & 0.3 \\
\hline
\end{tabular}




\begin{tabular}{|c|c|c|c|c|c|c|c|c|}
\hline \multicolumn{9}{|c|}{ Malate } \\
\hline $\begin{array}{c}\text { Observed } \\
\mathrm{m} / \mathrm{z}\end{array}$ & $\begin{array}{c}\text { Relative } \\
\text { Abundance }\end{array}$ & $\Delta \mathrm{m}$ & Chemical annotation & $\begin{array}{c}\text { Calculated } \\
\mathrm{m} / \mathrm{z}\end{array}$ & $\begin{array}{c}\text { Mass } \\
\text { accuracy } \\
\text { (ppm) }\end{array}$ & $\begin{array}{c}\text { Observed } \\
\mathrm{m} / \mathrm{z} \text { in } \\
{ }^{2} \mathrm{H}_{3}-\mathrm{Ac}\end{array}$ & $\begin{array}{c}\text { Calculated } \\
\mathrm{m} / \mathrm{z} \text { in } \\
{ }^{2} \mathrm{H}_{3}-\mathrm{Ac} \\
\end{array}$ & $\begin{array}{c}\text { Mass } \\
\text { accuracy in } \\
{ }^{2} \mathrm{H}_{3}-\mathrm{Ac}(\mathrm{ppm})\end{array}$ \\
\hline 72.9930 & $0.25 \%$ & -60.0212 & {$\left[\mathrm{M}-\mathrm{C}_{2} \mathrm{H}_{4} \mathrm{O}_{2}-\mathrm{H} \text { (Glyoxylate) }\right]^{-}$} & 72.9931 & -1.1 & 72.9931 & 72.9931 & -0.3 \\
\hline 89.0245 & $2.90 \%$ & -43.9898 & {$\left[\mathrm{M}-\mathrm{CO}_{2}-\mathrm{H}\right]^{-}$} & 89.0244 & 0.4 & 89.0243 & 89.0244 & -1.3 \\
\hline 115.0036 & $7.08 \%$ & -18.0106 & {$\left[\mathrm{M}-\mathrm{H}_{2} \mathrm{O}-\mathrm{H}\right]^{-}$} & 115.0037 & -0.7 & 115.0036 & 115.0037 & -0.7 \\
\hline 116.0072 & $0.24 \%$ & -17.0071 & $\left.{ }^{13} \mathrm{C}_{1}-\left[\mathrm{M}-\mathrm{H}_{2} \mathrm{O}-\mathrm{H}\right]\right]^{-}$ & 116.0070 & 1.0 & 116.0069 & 116.0070 & -1.2 \\
\hline 133.0142 & $100.00 \%$ & 0.0000 & {$[\mathrm{M}-\mathrm{H}]^{-}$} & 133.0143 & -0.2 & 133.0141 & 133.0143 & -1.1 \\
\hline 134.0175 & $4.61 \%$ & 1.0033 & ${ }^{13} \mathrm{C}_{1}-[\mathrm{M}-\mathrm{H}]^{-}$ & 134.0176 & -0.5 & 134.0175 & 134.0176 & -0.7 \\
\hline 135.0185 & $1.03 \%$ & 2.0043 & ${ }^{18} \mathrm{O}_{1}-[\mathrm{M}-\mathrm{H}]^{-}$ & 135.0185 & 0.4 & 135.0183 & 135.0185 & -1.4 \\
\hline 215.0188 & $0.29 \%$ & 82.0046 & {$[\mathrm{M}+\mathrm{NaAc}-\mathrm{H}]^{-}$} & 215.0173 & 6.9 & 218.0361 & 218.0362 & -0.2 \\
\hline 230.9822 & $2.02 \%$ & 97.9679 & {$[\mathrm{M}+\mathrm{CaAc}-2 \mathrm{H}]^{-}$} & 230.9823 & -0.7 & 234.0010 & 234.0012 & -0.6 \\
\hline 236.9997 & $0.26 \%$ & 103.9855 & {$\left[\mathrm{M}+\mathrm{Na}_{2} \mathrm{Ac}-2 \mathrm{H}\right]^{-}$} & 236.9993 & 1.7 & 240.0180 & 240.0181 & -0.4 \\
\hline 312.9855 & $0.70 \%$ & 179.9713 & {$\left[\mathrm{M}+\mathrm{NaCaAc} \mathrm{C}_{2}-2 \mathrm{H}\right]^{-}$} & 312.9854 & 0.4 & 319.0229 & 319.0231 & -0.5 \\
\hline 388.9717 & $0.39 \%$ & 255.9574 & {$\left[\mathrm{M}+\mathrm{Ca}_{2} \mathrm{Ac}_{3}-2 \mathrm{H}\right]^{-}$} & 388.9715 & 0.3 & 398.0280 & 398.0280 & 0.0 \\
\hline 394.9874 & $0.26 \%$ & 261.9732 & {$\left[\mathrm{M}+\mathrm{Na}_{2} \mathrm{CaAc}_{3}-2 \mathrm{H}\right]^{-}$} & 394.9885 & -2.7 & 404.0449 & 404.0450 & -0.1 \\
\hline 470.9745 & $0.23 \%$ & 337.9603 & {$\left[\mathrm{M}+\mathrm{NaCa}_{2} \mathrm{Ac}_{4}-2 \mathrm{H}\right]^{-}$} & 470.9746 & -0.2 & 483.0496 & 483.0499 & -0.6 \\
\hline
\end{tabular}




\begin{tabular}{|c|c|c|c|c|c|c|c|c|}
\hline \multicolumn{9}{|c|}{ Fructose6P } \\
\hline $\begin{array}{l}\text { Observed } \\
\mathrm{m} / \mathrm{z}\end{array}$ & $\begin{array}{c}\text { Relative } \\
\text { Abundance }\end{array}$ & $\Delta \mathrm{m}$ & Chemical annotation & $\begin{array}{c}\text { Calculated } \\
\mathrm{m} / \mathrm{z}\end{array}$ & $\begin{array}{c}\text { Mass } \\
\text { accuracy } \\
\text { (ppm) }\end{array}$ & $\begin{array}{c}\text { Observed } \\
\mathrm{m} / \mathrm{z} \text { in } \\
{ }^{2} \mathrm{H}_{3}-\mathrm{Ac}\end{array}$ & $\begin{array}{c}\text { Calculated } \\
\mathrm{m} / \mathrm{z} \text { in } \\
{ }^{2} \mathrm{H}_{3}-\mathrm{Ac} \\
\end{array}$ & $\begin{array}{c}\text { Mass } \\
\text { accuracy in } \\
{ }^{2} \mathrm{H}_{3}-\mathrm{Ac}(\mathrm{ppm})\end{array}$ \\
\hline 78.9588 & $2.23 \%$ & -180.0636 & {$\left[\mathrm{PO}_{3}\right]^{-}$} & 78.9591 & -3.3 & 78.9592 & 78.9591 & 1.9 \\
\hline 96.9694 & $4.54 \%$ & -162.0529 & {$\left[\mathrm{H}_{2} \mathrm{PO}_{4}\right]^{-}$} & 96.9696 & -2.0 & 96.9696 & 96.9696 & -0.2 \\
\hline 190.9731 & $1.29 \%$ & -68.0493 & $\begin{array}{c}{\left[\mathrm{M}-\mathrm{C}_{3} \mathrm{H}_{6} \mathrm{O}_{3}+\mathrm{Na}-2 \mathrm{H}\right.} \\
\text { (Glyceraldehyde phosphate) }]^{-}\end{array}$ & 190.9727 & 1.9 & 190.9727 & 190.9727 & 0.1 \\
\hline 200.9562 & $1.24 \%$ & -58.0662 & {$\left[\mathrm{HPO}_{4}+\mathrm{Na}_{2} \mathrm{Ac}\right]^{-}$} & 200.9546 & 7.7 & 203.9734 & 203.9735 & -0.3 \\
\hline 259.0223 & $100.00 \%$ & 0.0000 & {$[\mathrm{M}-\mathrm{H}]^{-}$} & 259.0224 & -0.4 & 259.0223 & 259.0224 & -0.5 \\
\hline 260.0260 & $6.73 \%$ & 1.0036 & ${ }^{13} \mathrm{C}_{1}-[\mathrm{M}-\mathrm{H}]^{-}$ & 260.0258 & 0.7 & 260.0256 & 260.0258 & -0.8 \\
\hline 261.0263 & $1.72 \%$ & 2.0040 & ${ }^{18} \mathrm{O}_{1-}-[\mathrm{M}-\mathrm{H}]^{-}$ & 261.0267 & -1.3 & 261.0265 & 261.0267 & -0.7 \\
\hline 266.9591 & $0.73 \%$ & 7.9368 & $\begin{array}{c}{\left[\mathrm{M}-\mathrm{C}_{3} \mathrm{H}_{6} \mathrm{O}_{3}+\mathrm{CaAc}-2 \mathrm{H}\right.} \\
\text { (Glyceraldehyde phosphate)] }\end{array}$ & 266.9588 & 1.1 & 269.9776 & 269.9777 & -0.2 \\
\hline 281.0046 & $4.13 \%$ & 21.9822 & {$[\mathrm{M}+\mathrm{Na}-2 \mathrm{H}]^{-}$} & 281.0044 & 0.6 & 281.0043 & 281.0044 & -0.3 \\
\hline 336.9998 & $2.11 \%$ & 77.9774 & {$\left[\mathrm{M}+\mathrm{H}_{2} \mathrm{SiO}_{3}-\mathrm{H}\right]^{-}$} & 336.9998 & 0.0 & 336.9998 & 336.9998 & 0.1 \\
\hline 341.0254 & $5.53 \%$ & 82.0030 & {$[\mathrm{M}+\mathrm{NaAc}-\mathrm{H}]^{-}$} & 341.0255 & -0.5 & 344.0443 & 344.0444 & -0.1 \\
\hline 356.9910 & $3.96 \%$ & 97.9686 & {$[\mathrm{M}+\mathrm{CaAc}-2 \mathrm{H}]^{-}$} & 356.9905 & 1.3 & 360.0090 & 360.0093 & -0.9 \\
\hline 363.0084 & $1.14 \%$ & 103.9861 & {$\left[\mathrm{M}+\mathrm{Na}_{2} \mathrm{Ac}-2 \mathrm{H}\right]^{-}$} & 363.0075 & 2.6 & 366.0262 & 366.0263 & -0.2 \\
\hline 438.9940 & $2.64 \%$ & 179.9716 & {$\left[\mathrm{M}+\mathrm{NaCaAc} \mathrm{C}_{2}-2 \mathrm{H}\right]^{-}$} & 438.9936 & 0.8 & 445.0311 & 445.0313 & -0.3 \\
\hline 445.0113 & $1.16 \%$ & 185.9889 & {$\left[\mathrm{M}+\mathrm{Na}_{3} \mathrm{Ac}_{2}-2 \mathrm{H}\right]^{-}$} & 445.0105 & 1.6 & 451.0483 & 451.0482 & 0.2 \\
\hline 520.9952 & $1.15 \%$ & 261.9728 & {$\left[\mathrm{M}+\mathrm{Na}_{2} \mathrm{CaAc}_{3}-2 \mathrm{H}\right]^{-}$} & 520.9967 & -2.8 & 530.0535 & 530.0532 & 0.7 \\
\hline 527.0133 & $0.80 \%$ & 267.9910 & {$\left[\mathrm{M}+\mathrm{Na}_{4} \mathrm{Ac}_{3}-2 \mathrm{H}\right]^{-}$} & 527.0136 & -0.6 & 536.0708 & 536.0701 & 1.3 \\
\hline 542.9778 & $1.25 \%$ & 283.9554 & {$\left[\mathrm{M}+\mathrm{Na}_{3} \mathrm{CaAc}_{3}-3 \mathrm{H}\right]^{-}$} & 542.9786 & -1.5 & 552.0352 & 552.0351 & 0.2 \\
\hline 618.9691 & $1.14 \%$ & 359.9468 & {$\left[\mathrm{M}+\mathrm{Na}_{2} \mathrm{Ca}_{2} \mathrm{Ac}_{4}-3 \mathrm{H}\right]^{-}$} & 618.9647 & 7.1 & 631.0406 & 631.0401 & 0.9 \\
\hline 624.9832 & $1.15 \%$ & 365.9609 & {$\left[\mathrm{M}+\mathrm{Na}_{4} \mathrm{CaAc}_{4}-3 \mathrm{H}\right]^{-}$} & 624.9817 & 2.5 & 637.0576 & 637.0570 & 0.9 \\
\hline
\end{tabular}




\begin{tabular}{|c|c|c|c|c|c|c|c|c|}
\hline \multicolumn{9}{|c|}{ Glucose6P } \\
\hline $\begin{array}{c}\text { Observed } \\
\mathrm{m} / \mathrm{z}\end{array}$ & $\begin{array}{l}\text { Relative } \\
\text { Abundance }\end{array}$ & $\Delta \mathrm{m}$ & Chemical annotation & $\begin{array}{c}\text { Calculated } \\
\mathrm{m} / \mathrm{z}\end{array}$ & $\begin{array}{c}\text { Mass } \\
\text { accuracy } \\
\text { (ppm) }\end{array}$ & $\begin{array}{c}\text { Observed } \\
\mathrm{m} / \mathrm{z} \text { in } \\
{ }^{2} \mathrm{H}_{3}-\mathrm{Ac}\end{array}$ & $\begin{array}{c}\text { Calculated } \\
\mathrm{m} / \mathrm{z} \text { in } \\
{ }^{2} \mathrm{H}_{3}-\mathrm{Ac}\end{array}$ & $\begin{array}{c}\text { Mass } \\
\text { accuracy in } \\
{ }^{2} \mathrm{H}_{3}-\mathrm{Ac}(\mathrm{ppm})\end{array}$ \\
\hline 78.9586 & $2.52 \%$ & -180.0637 & {$\left[\mathrm{PO}_{3}\right]^{-}$} & 78.9591 & -5.4 & 78.9591 & 78.9591 & 0.6 \\
\hline 96.9692 & $3.64 \%$ & -162.0531 & {$\left[\mathrm{H}_{2} \mathrm{PO}_{4}\right]^{-}$} & 96.9696 & -4.3 & 96.9696 & 96.9696 & -0.2 \\
\hline 160.9623 & $1.03 \%$ & -98.0600 & $\begin{array}{c}{\left[\mathrm{M}-\mathrm{C}_{4} \mathrm{H}_{8} \mathrm{O}_{4}+\mathrm{Na}-2 \mathrm{H}\right.} \\
\text { (Glycoaldehyde phosphate) }]^{-}\end{array}$ & 160.9621 & 1.1 & 160.9620 & 160.9621 & -0.8 \\
\hline 199.0020 & $0.85 \%$ & -60.0203 & $\begin{array}{c}{\left[\mathrm{M}-\mathrm{C}_{2} \mathrm{H}_{4} \mathrm{O}_{2}-\mathrm{H} \text { (Erythrose-4- }\right.} \\
\text { phospahte) }]^{-}\end{array}$ & 199.0013 & 3.7 & 199.0014 & 199.0013 & 0.5 \\
\hline 200.9543 & $0.65 \%$ & -58.0680 & {$\left[\mathrm{HPO}_{4}+\mathrm{Na}_{2} \mathrm{Ac}\right]^{-}$} & 200.9546 & -1.5 & 203.9733 & 203.9735 & -0.8 \\
\hline 236.9488 & $2.29 \%$ & -22.0735 & $\begin{array}{c}{\left[\mathrm{M}-\mathrm{C}_{4} \mathrm{H}_{8} \mathrm{O}_{4}+\mathrm{CaAc}-2 \mathrm{H}\right.} \\
\text { (Glycoaldehyde phosphate)] }\end{array}$ & 236.9483 & 2.4 & 239.9670 & 239.9671 & -0.3 \\
\hline 242.9651 & $0.41 \%$ & -16.0572 & $\begin{array}{c}{\left[\mathrm{M}-\mathrm{C}_{4} \mathrm{H}_{8} \mathrm{O}_{4}+\mathrm{Na}_{2} \mathrm{Ac}-2 \mathrm{H}\right.} \\
\text { (Glycoaldehyde phosphate) }]^{-}\end{array}$ & 242.9652 & -0.4 & 245.9839 & 245.9840 & -0.5 \\
\hline 259.0223 & $100.00 \%$ & 0.0000 & {$[\mathrm{M}-\mathrm{H}]^{-}$} & 259.0224 & -0.5 & 259.0224 & 259.0224 & -0.2 \\
\hline 260.0258 & $6.86 \%$ & 1.0034 & ${ }^{13} \mathrm{C}_{1}-[\mathrm{M}-\mathrm{H}]^{-}$ & 260.0258 & -0.2 & 260.0257 & 260.0258 & -0.4 \\
\hline 261.0262 & $1.86 \%$ & 2.0039 & ${ }^{18} \mathrm{O}_{1^{-}}[\mathrm{M}-\mathrm{H}]^{-}$ & 261.0267 & -1.8 & 261.0265 & 261.0267 & -0.7 \\
\hline 281.0042 & $2.37 \%$ & 21.9819 & {$[\mathrm{M}+\mathrm{Na}-2 \mathrm{H}]^{-}$} & 281.0044 & -0.5 & 281.0044 & 281.0044 & 0.0 \\
\hline 296.9700 & $0.37 \%$ & 37.9477 & $\begin{array}{c}{\left[\mathrm{M}-\mathrm{C}_{2} \mathrm{H}_{4} \mathrm{O}_{2}+\mathrm{CaAc}-2 \mathrm{H} \text { (Erythrose-4- }\right.} \\
\text { phospahte) }]^{-}\end{array}$ & 296.9694 & 2.2 & 299.9879 & 299.9882 & -1.0 \\
\hline 336.9999 & $1.15 \%$ & 77.9776 & {$\left[\mathrm{M}+\mathrm{H}_{2} \mathrm{SiO}_{3}-\mathrm{H}\right]^{-}$} & 336.9998 & 0.5 & 336.9998 & 336.9998 & 0.1 \\
\hline 341.0255 & $7.61 \%$ & 82.0032 & {$[\mathrm{M}+\mathrm{NaAc}-\mathrm{H}]^{-}$} & 341.0255 & 0.0 & 344.0443 & 344.0444 & -0.1 \\
\hline 342.0284 & $0.58 \%$ & 83.0061 & ${ }^{13} \mathrm{C}_{1}-[\mathrm{M}+\mathrm{NaAc}-\mathrm{H}]^{-}$ & 342.0289 & -1.3 & 345.0478 & 345.0477 & 0.3 \\
\hline 356.9909 & $2.84 \%$ & 97.9686 & {$[\mathrm{M}+\mathrm{CaAc}-2 \mathrm{H}]^{-}$} & 356.9905 & 1.2 & 360.0091 & 360.0093 & -0.7 \\
\hline 363.0078 & $0.82 \%$ & 103.9855 & {$\left[\mathrm{M}+\mathrm{Na}_{2} \mathrm{Ac}-2 \mathrm{H}\right]^{-}$} & 363.0075 & 1.0 & 366.0261 & 366.0263 & -0.5 \\
\hline 423.0287 & $0.87 \%$ & 164.0064 & {$\left[\mathrm{M}+\mathrm{Na}_{2} \mathrm{Ac}_{2}-\mathrm{H}\right]^{-}$} & 423.0286 & 0.2 & 429.0661 & 429.0663 & -0.3 \\
\hline 438.9943 & $1.68 \%$ & 179.9720 & {$\left[\mathrm{M}+\mathrm{NaCaAc} c_{2}-2 \mathrm{H}\right]^{-}$} & 438.9936 & 1.6 & 445.0309 & 445.0313 & -0.8 \\
\hline 445.0144 & $0.91 \%$ & 185.9921 & {$\left[\mathrm{M}+\mathrm{Na}_{3} \mathrm{Ac}_{2}-2 \mathrm{H}\right]^{-}$} & 445.0105 & 8.8 & 451.0481 & 451.0482 & -0.2 \\
\hline 460.9795 & $0.58 \%$ & 201.9572 & {$\left[\mathrm{M}+\mathrm{Na}_{2} \mathrm{CaAC}_{2}-3 \mathrm{H}\right]^{-}$} & 460.9755 & 8.6 & 467.0136 & 467.0132 & 0.9 \\
\hline 514.9810 & $0.30 \%$ & 255.9587 & {$\left[\mathrm{M}+\mathrm{Ca}_{2} \mathrm{Ac}_{3}-2 \mathrm{H}\right]^{-}$} & 514.9797 & 2.5 & 524.0366 & 524.0362 & 0.8 \\
\hline 519.0529 & $1.21 \%$ & 260.0306 & {$[2 \mathrm{M}-\mathrm{H}]^{-}$} & 519.0522 & 1.5 & 519.0521 & 519.0522 & -0.1 \\
\hline
\end{tabular}




\begin{tabular}{|c|c|c|c|c|c|c|c|c|}
\hline \multicolumn{9}{|c|}{ Glucose6P (cont'd) } \\
\hline $\begin{array}{c}\text { Observed } \\
\mathrm{m} / \mathrm{z}\end{array}$ & $\begin{array}{c}\text { Relative } \\
\text { Abundance }\end{array}$ & $\Delta \mathrm{m}$ & Chemical annotation & $\begin{array}{c}\text { Calculated } \\
\mathrm{m} / \mathrm{z}\end{array}$ & $\begin{array}{c}\text { Mass } \\
\text { accuracy } \\
(\mathrm{ppm})\end{array}$ & $\begin{array}{c}\text { Observed } \\
\mathrm{m} / \mathrm{z} \text { in } \\
{ }^{2} \mathrm{H}_{3}-\mathrm{Ac}\end{array}$ & $\begin{array}{c}\text { Calculated } \\
m / z \text { in } \\
{ }^{2} \mathrm{H}_{3}-\mathrm{Ac}\end{array}$ & $\begin{array}{c}\text { Mass } \\
\text { accuracy in } \\
{ }^{2} \mathrm{H}_{3}-\mathrm{Ac}(\mathrm{ppm})\end{array}$ \\
\hline 520.9959 & $0.75 \%$ & 261.9735 & {$[\mathrm{M}+\mathrm{Na} 2 \mathrm{CaAc} 3-2 \mathrm{H}]^{-}$} & 520.9967 & -1.6 & 530.0529 & 530.0532 & -0.5 \\
\hline 527.0125 & $0.60 \%$ & 267.9902 & {$[\mathrm{M}+\mathrm{Na} 4 \mathrm{Ac3}-2 \mathrm{H}]^{-}$} & 527.0136 & -2.1 & 536.0703 & 536.0701 & 0.4 \\
\hline 541.0364 & $0.58 \%$ & 282.0141 & {$[2 \mathrm{M}+\mathrm{Na}-2 \mathrm{H}]^{-}$} & 541.0341 & 4.2 & 541.0340 & 541.0341 & -0.2 \\
\hline 602.9961 & $0.48 \%$ & 343.9738 & {$[\mathrm{M}+\mathrm{Na} 3 \mathrm{CaAc4}-2 \mathrm{H}]^{-}$} & 602.9997 & -6.0 & 615.0758 & 615.0751 & 1.2 \\
\hline \multicolumn{9}{|c|}{ Fructose-1,6-bisphosphate } \\
\hline $\begin{array}{c}\text { Observed } \\
\mathrm{m} / \mathrm{z}\end{array}$ & $\begin{array}{c}\text { Relative } \\
\text { Abundance }\end{array}$ & $\Delta \mathrm{m}$ & Chemical annotation & $\begin{array}{c}\text { Calculated } \\
\mathrm{m} / \mathrm{z}\end{array}$ & $\begin{array}{c}\text { Mass } \\
\text { accuracy } \\
\text { (ppm) }\end{array}$ & $\begin{array}{c}\text { Observed } \\
\mathrm{m} / \mathrm{z} \text { in } \\
{ }^{2} \mathrm{H}_{3}-\mathrm{Ac} \\
\end{array}$ & $\begin{array}{c}\text { Calculated } \\
\mathrm{m} / \mathrm{z} \text { in } \\
{ }^{2} \mathrm{H}_{3}-\mathrm{Ac}\end{array}$ & $\begin{array}{c}\text { Mass } \\
\text { accuracy in } \\
{ }^{2} \mathrm{H}_{3}-\mathrm{Ac}(\mathrm{ppm})\end{array}$ \\
\hline 338.9888 & $100.00 \%$ & 0.0000 & {$[\mathrm{M}-\mathrm{H}]^{-}$} & 338.9888 & 0.1 & 338.9886 & 338.9888 & -0.5 \\
\hline 339.9924 & $6.74 \%$ & 1.0036 & ${ }^{13} \mathrm{C}_{1}-[\mathrm{M}-\mathrm{H}]^{-}$ & 339.9921 & 0.8 & 339.9921 & 339.9921 & -0.1 \\
\hline 340.9930 & $2.04 \%$ & 2.0042 & ${ }^{18} \mathrm{O}_{1}-[\mathrm{M}-\mathrm{H}]^{-}$ & 340.9930 & -0.1 & 340.9929 & 340.9930 & -0.4 \\
\hline 360.9706 & $16.05 \%$ & 21.9818 & {$[\mathrm{M}+\mathrm{Na}-2 \mathrm{H}]^{-}$} & 360.9707 & -0.3 & 360.9706 & 360.9707 & -0.3 \\
\hline 361.9740 & $0.66 \%$ & 22.9852 & ${ }^{13} \mathrm{C}_{1}-[\mathrm{M}+\mathrm{Na}-2 \mathrm{H}]^{-}$ & 361.9741 & -0.2 & 361.9741 & 361.9741 & 0.1 \\
\hline 376.9399 & $0.76 \%$ & 37.9511 & {$[\mathrm{M}+\mathrm{K}-2 \mathrm{H}]^{-}$} & 376.9447 & -12.6 & 376.9458 & 376.9447 & 3.1 \\
\hline 436.9570 & $5.28 \%$ & 97.9682 & {$[\mathrm{M}+\mathrm{CaAc}-2 \mathrm{H}]^{-}$} & 436.9568 & 0.4 & 439.9756 & 439.9757 & -0.2 \\
\hline 442.9750 & $3.28 \%$ & 103.9862 & {$\left[\mathrm{M}+\mathrm{Na}_{2} \mathrm{Ac}-2 \mathrm{H}\right]^{-}$} & 442.9738 & 2.7 & 445.9927 & 445.9926 & 0.2 \\
\hline 458.9390 & $1.85 \%$ & 119.9502 & {$[\mathrm{M}+\mathrm{NaCaAc}-3 \mathrm{H}]^{-}$} & 458.9388 & 0.5 & 461.9574 & 461.9576 & -0.5 \\
\hline 464.9573 & $1.44 \%$ & 125.9685 & {$\left[\mathrm{M}+\mathrm{Na}_{3} \mathrm{Ac}-3 \mathrm{H}\right]^{-}$} & 464.9557 & 3.4 & 467.9742 & 467.9746 & -0.8 \\
\hline 518.9615 & $1.07 \%$ & 179.9727 & {$\left[\mathrm{M}+\mathrm{NaCaAc} c_{2}-2 \mathrm{H}\right]^{-}$} & 518.9599 & 3.0 & 524.9979 & 524.9976 & 0.6 \\
\hline 540.9389 & $1.51 \%$ & 201.9501 & {$\left[\mathrm{M}+\mathrm{Na}_{2} \mathrm{CaAc}_{2}-3 \mathrm{H}\right]^{-}$} & 540.9419 & -5.5 & 546.9793 & 546.9795 & -0.4 \\
\hline 622.9448 & $1.10 \%$ & 283.9560 & {$\left[\mathrm{M}+\mathrm{Na}_{3} \mathrm{CaAc}_{3}-3 \mathrm{H}\right]^{-}$} & 622.9449 & -0.2 & 632.0025 & 632.0014 & 1.7 \\
\hline
\end{tabular}




\begin{tabular}{|c|c|c|c|c|c|c|c|c|}
\hline \multicolumn{9}{|c|}{ NAD } \\
\hline $\begin{array}{l}\text { Observed } \\
\mathrm{m} / \mathrm{z}\end{array}$ & $\begin{array}{l}\text { Relative } \\
\text { Abundance }\end{array}$ & $\Delta \mathrm{m}$ & Chemical annotation & $\begin{array}{c}\text { Calculated } \\
\mathrm{m} / \mathrm{z}\end{array}$ & $\begin{array}{c}\text { Mass } \\
\text { accuracy } \\
\text { (ppm) }\end{array}$ & $\begin{array}{c}\text { Observed } \\
\mathrm{m} / \mathrm{z} \text { in } \\
{ }^{2} \mathrm{H}_{3}-\mathrm{Ac}\end{array}$ & $\begin{array}{c}\text { Calculated } \\
\mathrm{m} / \mathrm{z} \text { in } \\
{ }^{2} \mathrm{H}_{3}-\mathrm{Ac}\end{array}$ & $\begin{array}{c}\text { Mass } \\
\text { accuracy in } \\
{ }^{2} \mathrm{H}_{3}-\mathrm{Ac}(\mathrm{ppm})\end{array}$ \\
\hline 540.0534 & $41.91 \%$ & -122.0483 & $\begin{array}{c}\text { [M-Nicotinamide-H (Cyclic ADP } \\
\text { Ribose) }]^{-}\end{array}$ & 540.0538 & -0.8 & 540.0538 & 540.0538 & -0.1 \\
\hline 541.0565 & $7.67 \%$ & -121.0452 & $\begin{array}{c}{ }^{13} \mathrm{C}_{1}-[\text { M-Nicotinamide-H (Cyclic } \\
\text { ADP Ribose) }]^{-}\end{array}$ & 541.0572 & -1.3 & 541.0574 & 541.0572 & 0.4 \\
\hline 542.0591 & $1.64 \%$ & -120.0426 & $\begin{array}{c}{ }^{18} \mathrm{O}_{1} \text {-[M-Nicotinamide-H (Cyclic } \\
\text { ADP Ribose) }]^{-}\end{array}$ & 542.0581 & 1.9 & 542.0580 & 542.0581 & -0.1 \\
\hline 562.0356 & $3.13 \%$ & -100.0661 & [M-Nicotinamide+Na-2H] ${ }^{-}$ & 562.0358 & -0.3 & 562.0361 & 562.0358 & 0.6 \\
\hline 638.0212 & $1.32 \%$ & -24.0805 & [M-Nicotinamide+CaAc-2H]- & 638.0219 & -1.1 & 641.0416 & 641.0407 & 1.4 \\
\hline 644.0386 & $1.62 \%$ & -18.0631 & [M-Nicotinamide $\left.+\mathrm{Na}_{2} \mathrm{Ac}-2 \mathrm{H}\right]^{-}$ & 644.0389 & -0.4 & 647.0581 & 647.0577 & 0.6 \\
\hline 662.1017 & $100.00 \%$ & 0.0000 & {$[\mathrm{M}-\mathrm{H}]^{-}$} & 662.1018 & -0.2 & 662.1019 & 662.1018 & 0.1 \\
\hline 663.1044 & $26.34 \%$ & 1.0027 & ${ }^{13} \mathrm{C}_{1}-[\mathrm{M}-\mathrm{H}]^{-}$ & 663.1052 & -1.2 & 663.1049 & 663.1052 & -0.5 \\
\hline 664.1068 & $5.86 \%$ & 2.0051 & ${ }^{18} \mathrm{O}_{1}-[\mathrm{M}-\mathrm{H}]^{-}$ & 664.1061 & 1.1 & 664.1072 & 664.1061 & 1.7 \\
\hline 665.1110 & $0.88 \%$ & 3.0093 & $\left.{ }^{13} \mathrm{C}_{1}{ }^{18} \mathrm{O}_{1}-[\mathrm{M}-\mathrm{H}]\right]^{-}$ & 665.1095 & 2.3 & 665.1091 & 665.1095 & -0.5 \\
\hline 684.0855 & $2.93 \%$ & 21.9838 & {$[\mathrm{M}+\mathrm{Na}-2 \mathrm{H}]^{-}$} & 684.0838 & 2.5 & 684.0854 & 684.0838 & 2.4 \\
\hline 685.0887 & $0.55 \%$ & 22.9870 & ${ }^{13} \mathrm{C}_{1}-[\mathrm{M}+\mathrm{Na}-2 \mathrm{H}]^{-}$ & 685.0871 & 2.3 & 685.0880 & 685.0871 & 1.3 \\
\hline 720.0222 & $1.25 \%$ & 57.9205 & [M-Nicotinamide+NaCaAc $2-2 \mathrm{H}]^{-}$ & 720.0250 & -3.9 & 726.0642 & 726.0626 & 2.1 \\
\hline 724.0753 & $0.71 \%$ & 61.9736 & {$\left[\mathrm{M}+\mathrm{Na}_{2}(\mathrm{OH})-2 \mathrm{H}\right]^{-}$} & 724.0763 & -1.4 & 724.0777 & 724.0763 & 1.9 \\
\hline 744.1055 & $12.04 \%$ & 82.0038 & {$[\mathrm{M}+\mathrm{NaAc}-\mathrm{H}]^{-}$} & 744.1049 & 0.8 & 747.1247 & 747.1238 & 1.3 \\
\hline 745.1094 & $3.36 \%$ & 83.0077 & ${ }^{13} \mathrm{C}_{1}-[\mathrm{M}+\mathrm{NaAc}-\mathrm{H}]^{-}$ & 745.1083 & 1.5 & 748.1296 & 748.1271 & 3.3 \\
\hline 746.1118 & $0.70 \%$ & 84.0101 & ${ }^{18} \mathrm{O}_{1^{-}}[\mathrm{M}+\mathrm{NaAc}-\mathrm{H}]^{-}$ & 746.1092 & 3.5 & 749.1318 & 749.1280 & 5.1 \\
\hline 760.0731 & $3.02 \%$ & 97.9714 & {$[\mathrm{M}+\mathrm{CaAc}-2 \mathrm{H}]^{-}$} & 760.0699 & 4.2 & 763.0927 & 763.0888 & 5.2 \\
\hline 761.0754 & $0.69 \%$ & 98.9737 & ${ }^{13} \mathrm{C}_{1}-[\mathrm{M}+\mathrm{CaAc}-2 \mathrm{H}]^{-}$ & 761.0733 & 2.8 & 764.0950 & 764.0921 & 3.8 \\
\hline 784.1068 & $0.79 \%$ & 122.0051 & {$\left[\mathrm{M}+\mathrm{Na}_{2}(\mathrm{OH}) \mathrm{Ac}-\mathrm{H}\right]^{-}$} & 784.0974 & 12.0 & 787.1172 & 787.1163 & 1.2 \\
\hline 820.0956 & $2.99 \%$ & 157.9939 & {$\left[\mathrm{M}+\mathrm{CaAc} \mathrm{C}_{2}-\mathrm{H}\right]^{-}$} & 820.0910 & 5.6 & 826.1310 & 826.1287 & 2.8 \\
\hline 821.0914 & $0.71 \%$ & 158.9897 & ${ }^{13} \mathrm{C}_{1}-\left[\mathrm{M}+\mathrm{CaAc} \mathrm{C}_{2}-\mathrm{H}\right]^{-}$ & 821.0944 & -3.7 & 827.1349 & 827.1321 & 3.4 \\
\hline 902.0943 & $0.54 \%$ & 239.9926 & {$\left[\mathrm{M}+\mathrm{NaCaAc} c_{3}-\mathrm{H}\right]^{-}$} & 902.0941 & 0.2 & 911.1532 & 911.1506 & 2.8 \\
\hline
\end{tabular}




\begin{tabular}{|c|c|c|c|c|c|c|c|c|}
\hline \multicolumn{9}{|c|}{ ATP } \\
\hline $\begin{array}{c}\text { Observed } \\
\mathrm{m} / \mathrm{z}\end{array}$ & $\begin{array}{c}\text { Relative } \\
\text { Abundance }\end{array}$ & $\Delta \mathrm{m}$ & Chemical annotation & $\begin{array}{c}\text { Calculated } \\
\mathrm{m} / \mathrm{z}\end{array}$ & $\begin{array}{c}\text { Mass } \\
\text { accuracy } \\
\text { (ppm) }\end{array}$ & $\begin{array}{c}\text { Observed } \\
\mathrm{m} / \mathrm{z} \text { in } \\
{ }^{2} \mathrm{H}_{3}-\mathrm{Ac}\end{array}$ & $\begin{array}{c}\text { Calculated } \\
\mathrm{m} / \mathrm{z} \text { in } \\
{ }^{2} \mathrm{H}_{3}-\mathrm{Ac} \\
\end{array}$ & $\begin{array}{c}\text { Mass } \\
\text { accuracy in } \\
{ }^{2} \mathrm{H}_{3}-\mathrm{Ac}(\mathrm{ppm})\end{array}$ \\
\hline 78.9591 & $3.56 \%$ & -427.0295 & {$\left[\mathrm{PO}_{3}\right]^{-}$} & 78.9591 & 0.0 & 78.9591 & 78.9591 & 0.6 \\
\hline 263.4750 & $5.24 \%$ & -242.5136 & {$[\mathrm{M}+\mathrm{Mg}-4 \mathrm{H}]^{2-}$} & 263.4753 & -1.2 & 263.4749 & 263.4753 & -1.5 \\
\hline 263.9764 & $1.04 \%$ & -242.0122 & $\left.{ }^{13} \mathrm{C}_{1}-[\mathrm{M}+\mathrm{Mg}-4 \mathrm{H}]\right]^{2-}$ & 263.9757 & 2.5 & 263.9763 & 263.9757 & 2.3 \\
\hline 293.4853 & $1.48 \%$ & -212.5033 & {$[\mathrm{M}+\mathrm{MgAc}-3 \mathrm{H}]^{2-}$} & 293.4859 & -2.0 & 294.9952 & 294.9953 & -0.3 \\
\hline 301.4747 & $8.71 \%$ & -204.5139 & {$[\mathrm{M}+\mathrm{CaAc}-3 \mathrm{H}]^{2-}$} & 301.4746 & 0.2 & 302.9840 & 302.9840 & -0.1 \\
\hline 301.9761 & $1.76 \%$ & -204.0125 & ${ }^{13} \mathrm{C}_{1}-[\mathrm{M}+\mathrm{CaAc}-3 \mathrm{H}]^{2-}$ & 301.9763 & -0.7 & 303.4856 & 303.4857 & -0.4 \\
\hline 350.4598 & $1.89 \%$ & -155.5288 & {$\left[\mathrm{M}+\mathrm{Ca}_{2} \mathrm{Ac}_{2}-4 \mathrm{H}\right]^{2-}$} & 350.4587 & 3.1 & 353.4774 & 353.4775 & -0.3 \\
\hline 426.0219 & $8.86 \%$ & -79.9667 & {$\left[\mathrm{M}-\mathrm{HPO}_{3}-\mathrm{H}(\mathrm{ADP})\right]^{-}$} & 426.0221 & -0.6 & 426.0220 & 426.0221 & -0.3 \\
\hline 443.4528 & $1.23 \%$ & -62.5358 & {$\left[\mathrm{M}+\mathrm{Na}_{3} \mathrm{Ca}_{2} \mathrm{Ac}_{4}-5 \mathrm{H}\right]^{2-}$} & 443.4527 & 0.3 & 449.4896 & 449.4904 & -1.7 \\
\hline 505.9886 & $100.00 \%$ & 0.0000 & {$[\mathrm{M}-\mathrm{H}]^{-}$} & 505.9885 & 0.2 & 505.9884 & 505.9885 & -0.1 \\
\hline 506.9911 & $13.12 \%$ & 1.0025 & ${ }^{13} \mathrm{C}_{1}-[\mathrm{M}-\mathrm{H}]^{-}$ & 506.9918 & -1.4 & 506.9920 & 506.9918 & 0.4 \\
\hline 507.9930 & $3.20 \%$ & 2.0044 & ${ }^{18} \mathrm{O}_{1}-[\mathrm{M}-\mathrm{H}]^{-}$ & 507.9927 & 0.5 & 507.9927 & 507.9927 & 0.0 \\
\hline 527.9701 & $17.21 \%$ & 21.9815 & {$[\mathrm{M}+\mathrm{Na}-2 \mathrm{H}]^{-}$} & 527.9704 & -0.6 & 527.9703 & 527.9704 & -0.2 \\
\hline 528.9738 & $2.15 \%$ & 22.9852 & ${ }^{13} \mathrm{C}_{1}-[\mathrm{M}+\mathrm{Na}-2 \mathrm{H}]^{-}$ & 528.9738 & 0.0 & 528.9736 & 528.9738 & -0.3 \\
\hline 549.9506 & $4.17 \%$ & 43.9620 & {$[\mathrm{M}+2 \mathrm{Na}-3 \mathrm{H}]^{-}$} & 549.9524 & -3.3 & 549.9521 & 549.9524 & -0.5 \\
\hline 587.9815 & $5.16 \%$ & 81.9929 & {$[\mathrm{M}+\mathrm{MgAc}-2 \mathrm{H}]^{-}$} & 587.9790 & 4.3 & 590.9982 & 590.9978 & 0.6 \\
\hline 588.9833 & $1.38 \%$ & 82.9947 & ${ }^{13} \mathrm{C}_{1}-[\mathrm{M}+\mathrm{MgAc}-2 \mathrm{H}]^{-}$ & 588.9823 & 1.6 & $N^{*}$ & 592.0012 & $N D^{*}$ \\
\hline 603.9564 & $13.42 \%$ & 97.9678 & {$[\mathrm{M}+\mathrm{CaAc}-2 \mathrm{H}]^{-}$} & 603.9565 & -0.3 & 606.9753 & 606.9754 & -0.1 \\
\hline 604.9559 & $2.00 \%$ & 98.9673 & ${ }^{13} \mathrm{C}_{1}-[\mathrm{M}+\mathrm{CaAc}-2 \mathrm{H}]^{-}$ & 604.9599 & -6.6 & 607.9781 & 607.9787 & -1.0 \\
\hline 609.9582 & $1.46 \%$ & 103.9696 & {$[\mathrm{M}+\mathrm{NaMgAc}-3 \mathrm{H}]^{-}$} & 609.9609 & -4.5 & $N^{*}$ & 612.9798 & $\mathrm{ND}^{*}$ \\
\hline 625.9389 & $7.48 \%$ & 119.9503 & {$[\mathrm{M}+\mathrm{NaCaAc}-3 \mathrm{H}]^{-}$} & 625.9385 & 0.7 & 628.9575 & 628.9573 & 0.3 \\
\hline 631.9533 & $1.27 \%$ & 125.9647 & {$\left[\mathrm{M}+\mathrm{Na}_{3} \mathrm{Ac}-3 \mathrm{H}\right]^{-}$} & 631.9554 & -3.4 & 634.9735 & 634.9743 & -1.2 \\
\hline 707.9443 & $3.17 \%$ & 201.9558 & {$\left[\mathrm{M}+\mathrm{Na}_{2} \mathrm{CaAc}_{2}-3 \mathrm{H}\right]^{-}$} & 707.9416 & 3.9 & 713.9799 & 713.9792 & 1.0 \\
\hline 811.9288 & $0.91 \%$ & 305.9402 & {$\left[\mathrm{M}+\mathrm{Na}_{4} \mathrm{CaAc}_{3}-4 \mathrm{H}\right]^{-}$} & 811.9266 & 2.7 & 820.9862 & 820.9831 & 3.8 \\
\hline
\end{tabular}


Table S2. The rank correlation coefficients and the ranks scores for Glc6P and NAD calculated by InCIDR

\begin{tabular}{|c|c|c|c|c|c|c|c|c|c|c|c|}
\hline \multicolumn{12}{|c|}{ Glucose6P } \\
\hline Observed & & & & & & & & & & Rank & \\
\hline $\mathrm{mz}$ & CID_0eV & CID_2eV & CID_4eV & CID_6eV & CID_8eV & CID_10eV & CID_15eV & CID_20eV & tau & Score & Annotation \\
\hline 78.9586 & $7.39 E+06$ & $7.84 \mathrm{E}+06$ & $8.18 \mathrm{E}+06$ & $9.76 \mathrm{E}+06$ & $1.15 \mathrm{E}+07$ & $1.32 \mathrm{E}+07$ & $2.01 \mathrm{E}+07$ & $2.77 \mathrm{E}+07$ & -1.000 & 10.563 & {$\left[\mathrm{PO}_{3}\right]^{-}$} \\
\hline 96.9692 & $1.07 \mathrm{E}+07$ & $1.25 \mathrm{E}+07$ & $1.57 \mathrm{E}+07$ & $2.32 \mathrm{E}+07$ & $3.23 \mathrm{E}+07$ & $4.50 \mathrm{E}+07$ & $8.09 E+07$ & $1.09 \mathrm{E}+08$ & -1.000 & 12.563 & {$\left[\mathrm{H}_{2} \mathrm{PO}_{4}\right]^{-}$} \\
\hline 160.9623 & $3.04 \mathrm{E}+06$ & $3.93 E+06$ & $5.22 \mathrm{E}+06$ & $6.78 \mathrm{E}+06$ & $8.91 E+06$ & $1.07 \mathrm{E}+07$ & $1.29 \mathrm{E}+07$ & $1.39 \mathrm{E}+07$ & -1.000 & 14.563 & {$\left[\mathrm{M}-\mathrm{C}_{4} \mathrm{H}_{8} \mathrm{O}_{4}+\mathrm{Na}-2 \mathrm{H}\right]^{-}$} \\
\hline 199.0020 & $2.51 E+06$ & $4.06 \mathrm{E}+06$ & $6.42 E+06$ & $1.05 E+07$ & $1.54 \mathrm{E}+07$ & $2.04 E+07$ & $2.71 E+07$ & $2.25 E+07$ & -0.929 & 16.563 & {$\left[\mathrm{M}-\mathrm{C}_{2} \mathrm{H}_{4} \mathrm{O}_{2}-\mathrm{H}\right]^{-}$} \\
\hline 200.9543 & $1.92 E+06$ & $2.29 E+06$ & $2.43 E+06$ & $3.04 \mathrm{E}+06$ & $3.33 \mathrm{E}+06$ & $3.53 E+06$ & $3.86 \mathrm{E}+06$ & $3.85 \mathrm{E}+06$ & -0.929 & 18.420 & {$\left[\mathrm{HPO}_{4}+\mathrm{Na}_{2} \mathrm{Ac}\right]^{-}$} \\
\hline 236.9488 & $6.71 E+06$ & $6.66 \mathrm{E}+06$ & $6.34 \mathrm{E}+06$ & $7.39 E+06$ & $7.93 E+06$ & $8.56 \mathrm{E}+06$ & $9.64 \mathrm{E}+06$ & $1.01 \mathrm{E}+07$ & -0.786 & 20.277 & {$\left[\mathrm{M}-\mathrm{C}_{4} \mathrm{H}_{8} \mathrm{O}_{4}+\mathrm{CaAc}-2 \mathrm{H}\right]^{-}$} \\
\hline 242.9651 & $1.20 \mathrm{E}+06$ & $1.58 \mathrm{E}+06$ & $1.41 \mathrm{E}+06$ & $1.83 E+06$ & $2.05 E+06$ & $2.41 E+06$ & $3.02 E+06$ & $3.29 \mathrm{E}+06$ & -0.929 & 21.848 & {$\left[\mathrm{M}-\mathrm{C}_{4} \mathrm{H}_{8} \mathrm{O}_{4}+\mathrm{Na}_{2} \mathrm{Ac}-2 \mathrm{H}\right]^{-}$} \\
\hline 259.0223 & $2.94 \mathrm{E}+08$ & $2.85 \mathrm{E}+08$ & $2.76 \mathrm{E}+08$ & $2.61 E+08$ & $2.36 \mathrm{E}+08$ & $2.02 E+08$ & $1.14 \mathrm{E}+08$ & $5.30 \mathrm{E}+07$ & 1.000 & 23.706 & {$[\mathrm{M}-\mathrm{H}]^{-}$} \\
\hline 260.0258 & $2.01 E+07$ & $1.95 \mathrm{E}+07$ & $1.87 \mathrm{E}+07$ & $1.73 E+07$ & $1.59 \mathrm{E}+07$ & $1.35 \mathrm{E}+07$ & $7.66 \mathrm{E}+06$ & $3.33 E+06$ & 1.000 & 21.706 & ${ }^{13} \mathrm{C}_{1}-[\mathrm{M}-\mathrm{H}]^{-}$ \\
\hline 261.0262 & $5.47 E+06$ & $5.37 E+06$ & $4.76 \mathrm{E}+06$ & $4.55 E+06$ & $4.34 \mathrm{E}+06$ & $3.65 E+06$ & $1.95 \mathrm{E}+06$ & $7.33 E+05$ & 1.000 & 19.706 & ${ }^{18} \mathrm{O}_{1}-[\mathrm{M}-\mathrm{H}]^{-}$ \\
\hline 281.0042 & $6.97 E+06$ & $8.30 E+06$ & $8.18 \mathrm{E}+06$ & $7.81 E+06$ & $6.53 E+06$ & $4.60 E+06$ & $1.23 E+06$ & $8.59 E+04$ & 0.786 & 17.706 & {$[\mathrm{M}+\mathrm{Na}-2 \mathrm{H}]^{-}$} \\
\hline 296.9700 & $1.09 E+06$ & 1.17E+06 & $7.54 \mathrm{E}+05$ & $7.63 E+05$ & $6.29 \mathrm{E}+05$ & $4.64 \mathrm{E}+05$ & $1.23 E+05$ & $0.00 E+00$ & 0.857 & 16.134 & {$[\mathrm{M}+\mathrm{Ca}-3 \mathrm{H}]^{-}$} \\
\hline 336.9999 & $3.37 E+06$ & $3.21 E+06$ & $2.96 \mathrm{E}+06$ & $2.67 E+06$ & $2.80 E+06$ & $2.22 E+06$ & $1.23 \mathrm{E}+06$ & $3.30 E+05$ & 0.929 & 14.420 & {$\left[\mathrm{M}+\mathrm{H}_{2} \mathrm{SiO}_{3}-\mathrm{H}\right]^{-}$} \\
\hline 341.0255 & $2.23 E+07$ & $1.81 \mathrm{E}+07$ & $1.34 \mathrm{E}+07$ & $9.30 E+06$ & $6.02 E+06$ & $3.20 \mathrm{E}+06$ & $2.18 \mathrm{E}+05$ & $1.73 E+04$ & 1.000 & 12.563 & {$[\mathrm{M}+\mathrm{NaAc}-\mathrm{H}]^{-}$} \\
\hline 342.0284 & $1.71 E+06$ & $1.38 \mathrm{E}+06$ & $7.21 E+05$ & $2.74 \mathrm{E}+05$ & $1.71 \mathrm{E}+05$ & $1.65 \mathrm{E}+04$ & $0.00 E+00$ & $0.00 \mathrm{E}+00$ & 0.982 & 10.563 & $\left.{ }^{13} \mathrm{C}_{1}-[\mathrm{M}+\mathrm{NaAc}-\mathrm{H}]\right]^{-}$ \\
\hline 356.9909 & $8.35 E+06$ & $7.15 E+06$ & $6.20 E+06$ & $4.57 E+06$ & $3.71 E+06$ & $2.55 E+06$ & $6.44 \mathrm{E}+05$ & $0.00 E+00$ & 1.000 & 8.599 & {$[\mathrm{M}+\mathrm{CaAc}-2 \mathrm{H}]^{-}$} \\
\hline 363.0078 & $2.40 \mathrm{E}+06$ & $2.43 E+06$ & $1.72 \mathrm{E}+06$ & $1.65 \mathrm{E}+06$ & $8.53 E+05$ & $2.96 \mathrm{E}+05$ & $0.00 \mathrm{E}+00$ & $0.00 \mathrm{E}+00$ & 0.909 & 6.599 & {$\left[\mathrm{M}+\mathrm{Na}_{2} \mathrm{Ac}-2 \mathrm{H}\right]^{-}$} \\
\hline 423.0287 & $2.55 E+06$ & $1.52 E+06$ & $4.94 \mathrm{E}+05$ & $1.95 \mathrm{E}+05$ & $0.00 E+00$ & $0.00 E+00$ & $0.00 E+00$ & $0.00 \mathrm{E}+00$ & 0.886 & 4.780 & {$\left[\mathrm{M}+\mathrm{Na}_{2} \mathrm{Ac}_{2}-\mathrm{H}\right]^{-}$} \\
\hline 438.9943 & $4.94 \mathrm{E}+06$ & 4.67E+06 & $3.98 \mathrm{E}+06$ & $2.86 \mathrm{E}+06$ & $2.07 E+06$ & $1.29 \mathrm{E}+06$ & $1.76 \mathrm{E}+05$ & $0.00 \mathrm{E}+00$ & 1.000 & 3.007 & {$\left[\mathrm{M}+\mathrm{NaCaAc}{ }_{2}-2 \mathrm{H}\right]^{-}$} \\
\hline 445.0144 & $2.66 \mathrm{E}+06$ & $2.43 E+06$ & $1.75 \mathrm{E}+06$ & $1.49 \mathrm{E}+06$ & $8.40 \mathrm{E}+05$ & $5.94 \mathrm{E}+05$ & $7.58 \mathrm{E}+04$ & $0.00 \mathrm{E}+00$ & 1.000 & 1.007 & {$\left[\mathrm{M}+\mathrm{Na}_{3} \mathrm{Ac}_{2}-2 \mathrm{H}\right]^{-}$} \\
\hline 460.9795 & $1.70 E+06$ & $2.06 \mathrm{E}+06$ & $1.28 \mathrm{E}+06$ & $1.48 \mathrm{E}+06$ & $1.22 \mathrm{E}+06$ & $9.32 E+05$ & $2.41 E+05$ & $7.70 \mathrm{E}+04$ & 0.857 & -0.993 & {$\left[\mathrm{M}+\mathrm{Na}_{2} \mathrm{CaAc}_{2}-3 \mathrm{H}\right]^{-}$} \\
\hline 514.9810 & $8.72 E+05$ & $5.74 \mathrm{E}+05$ & $3.85 E+05$ & $1.78 \mathrm{E}+05$ & $9.67 E+04$ & $7.37 E+04$ & $0.00 \mathrm{E}+00$ & $0.00 \mathrm{E}+00$ & 0.982 & -2.707 & {$\left[\mathrm{M}+\mathrm{Ca}_{2} \mathrm{Ac}_{3}-2 \mathrm{H}\right]^{-}$} \\
\hline 519.0529 & $3.55 E+06$ & $3.83 E+06$ & $3.59 E+06$ & $3.83 E+06$ & $3.56 \mathrm{E}+06$ & $3.25 E+06$ & $2.02 E+06$ & $9.25 E+05$ & 0.571 & -4.671 & {$[2 \mathrm{M}-\mathrm{H}]^{-}$} \\
\hline 520.9959 & $2.20 E+06$ & $1.78 \mathrm{E}+06$ & $1.12 \mathrm{E}+06$ & $7.36 \mathrm{E}+05$ & $3.24 \mathrm{E}+05$ & $1.60 \mathrm{E}+05$ & $0.00 E+00$ & $0.00 E+00$ & 0.982 & -5.814 & {$\left[\mathrm{M}+\mathrm{Na}_{2} \mathrm{CaAc}_{3}-2 \mathrm{H}\right]^{-}$} \\
\hline 527.0125 & $1.77 \mathrm{E}+06$ & $1.66 \mathrm{E}+06$ & $1.27 \mathrm{E}+06$ & $1.01 \mathrm{E}+06$ & $7.20 \mathrm{E}+05$ & $2.77 \mathrm{E}+05$ & $0.00 \mathrm{E}+00$ & $0.00 \mathrm{E}+00$ & 0.982 & -7.778 & {$\left[\mathrm{M}+\mathrm{Na}_{4} \mathrm{Ac}_{3}-2 \mathrm{H}\right]^{-}$} \\
\hline 541.0364 & $1.69 \mathrm{E}+06$ & $1.88 \mathrm{E}+06$ & $1.86 \mathrm{E}+06$ & $2.00 E+06$ & $1.96 \mathrm{E}+06$ & $1.89 \mathrm{E}+06$ & $2.08 \mathrm{E}+06$ & $1.97 E+06$ & -0.571 & -9.742 & {$[2 \mathrm{M}+\mathrm{Na}-2 \mathrm{H}]^{-}$} \\
\hline 602.9961 & $1.40 \mathrm{E}+06$ & $1.12 \mathrm{E}+06$ & $5.34 \mathrm{E}+05$ & $1.78 \mathrm{E}+05$ & $5.49 \mathrm{E}+04$ & $2.37 E+04$ & $0.00 \mathrm{E}+00$ & $0.00 \mathrm{E}+00$ & 0.982 & -8.599 & {$\left[\mathrm{M}+\mathrm{Na}_{3} \mathrm{CaAc}_{4}-2 \mathrm{H}\right]^{-}$} \\
\hline
\end{tabular}




\begin{tabular}{|c|c|c|c|c|c|c|c|c|c|c|c|}
\hline \multicolumn{12}{|c|}{ NAD } \\
\hline $\mathrm{mz}$ & CID_OeV & CID_2eV & CID_4eV & CID_6eV & CID_8eV & CID_10eV & CID_15eV & CID_20eV & tau & $\begin{array}{l}\text { Rank } \\
\text { Score }\end{array}$ & Annotation \\
\hline 540.0534 & $6.47 \mathrm{E}+07$ & $7.97 \mathrm{E}+07$ & $9.89 \mathrm{E}+07$ & $1.26 \mathrm{E}+08$ & $1.51 \mathrm{E}+08$ & $1.83 \mathrm{E}+08$ & $2.39 \mathrm{E}+08$ & $2.63 \mathrm{E}+08$ & -1.000 & 1.692 & [M-Nic-H]- \\
\hline 541.0565 & $1.19 \mathrm{E}+07$ & $1.46 \mathrm{E}+07$ & $1.86 \mathrm{E}+07$ & $2.34 \mathrm{E}+07$ & $2.84 \mathrm{E}+07$ & $3.40 \mathrm{E}+07$ & $4.45 \mathrm{E}+07$ & $4.87 \mathrm{E}+07$ & -1.000 & 3.692 & $\left.{ }^{13} \mathrm{C}_{1}-[\mathrm{M}-\mathrm{NiC}-\mathrm{H}]\right]^{-}$ \\
\hline 542.0591 & $2.54 \mathrm{E}+06$ & $3.16 \mathrm{E}+06$ & $3.98 \mathrm{E}+06$ & $5.46 \mathrm{E}+06$ & $6.51 \mathrm{E}+06$ & $7.90 \mathrm{E}+06$ & $1.00 \mathrm{E}+07$ & $1.13 \mathrm{E}+07$ & -1.000 & 5.692 & ${ }^{18} \mathrm{O}_{1}-[\mathrm{M}-\mathrm{Nic}-\mathrm{H}]^{-}$ \\
\hline 562.0356 & $4.84 \mathrm{E}+06$ & $5.39 E+06$ & $5.76 \mathrm{E}+06$ & $6.76 \mathrm{E}+06$ & $8.17 \mathrm{E}+06$ & $9.89 E+06$ & $1.74 \mathrm{E}+07$ & $2.70 \mathrm{E}+07$ & -1.000 & 7.692 & {$[\mathrm{M}-\mathrm{Nic}+\mathrm{Na}-2 \mathrm{H}]^{-}$} \\
\hline 638.0212 & $2.04 \mathrm{E}+06$ & $2.22 \mathrm{E}+06$ & $2.70 \mathrm{E}+06$ & $3.32 \mathrm{E}+06$ & $4.24 \mathrm{E}+06$ & $5.32 \mathrm{E}+06$ & $8.51 E+06$ & $1.20 \mathrm{E}+07$ & -1.000 & 9.692 & {$[\mathrm{M}-\mathrm{Nic}+\mathrm{CaAc}-2 \mathrm{H}]^{-}$} \\
\hline 644.0386 & $2.51 E+06$ & $2.68 \mathrm{E}+06$ & $2.99 \mathrm{E}+06$ & $3.06 \mathrm{E}+06$ & $3.24 \mathrm{E}+06$ & $3.90 E+06$ & $4.53 E+06$ & $5.15 E+06$ & -1.000 & 11.692 & {$\left[\mathrm{M}-\mathrm{Nic}+\mathrm{Na}_{2} \mathrm{Ac}-2 \mathrm{H}\right]^{-}$} \\
\hline 662.1017 & $1.54 \mathrm{E}+08$ & $1.49 \mathrm{E}+08$ & $1.39 \mathrm{E}+08$ & $1.20 \mathrm{E}+08$ & $9.43 E+07$ & $7.18 \mathrm{E}+07$ & $2.57 E+07$ & $7.39 E+06$ & 1.000 & 13.692 & {$[\mathrm{M}-\mathrm{H}]^{-}$} \\
\hline 663.1044 & $4.07 \mathrm{E}+07$ & $3.87 \mathrm{E}+07$ & $3.59 \mathrm{E}+07$ & $3.13 \mathrm{E}+07$ & $2.47 \mathrm{E}+07$ & $1.80 \mathrm{E}+07$ & $6.31 E+06$ & $1.70 \mathrm{E}+06$ & 1.000 & 11.692 & ${ }^{13} \mathrm{C}_{1}-[\mathrm{M}-\mathrm{H}]^{-}$ \\
\hline 664.1068 & $9.05 E+06$ & $8.66 \mathrm{E}+06$ & $7.84 \mathrm{E}+06$ & $7.29 E+06$ & $5.46 \mathrm{E}+06$ & 4.13E+06 & $1.23 \mathrm{E}+06$ & $1.32 \mathrm{E}+05$ & 1.000 & 9.692 & ${ }^{18} \mathrm{O}_{1}-[\mathrm{M}-\mathrm{H}]^{-}$ \\
\hline 665.1110 & $1.36 \mathrm{E}+06$ & $1.28 \mathrm{E}+06$ & $9.42 \mathrm{E}+05$ & $6.74 \mathrm{E}+05$ & $5.09 E+05$ & $4.72 \mathrm{E}+05$ & $0.00 E+00$ & $0.00 E+00$ & 0.982 & 7.692 & $\left.{ }^{13} \mathrm{C}_{1}{ }^{18} \mathrm{O}_{1}-[\mathrm{M}-\mathrm{H}]\right]^{-}$ \\
\hline 684.0855 & $4.52 E+06$ & $6.33 E+06$ & $8.25 E+06$ & $1.06 \mathrm{E}+07$ & $1.26 \mathrm{E}+07$ & $1.47 \mathrm{E}+07$ & $1.41 \mathrm{E}+07$ & $8.70 E+06$ & -0.643 & 5.728 & {$[\mathrm{M}+\mathrm{Na}-2 \mathrm{H}]^{-}$} \\
\hline 685.0887 & $8.51 E+05$ & $1.42 \mathrm{E}+06$ & 1.77E+06 & $2.53 E+06$ & $3.01 E+06$ & $3.85 E+06$ & $3.62 E+06$ & $2.00 E+06$ & -0.643 & 7.013 & $\left.{ }^{13} \mathrm{C}_{1}-[\mathrm{M}+\mathrm{Na}-2 \mathrm{H}]\right]^{-}$ \\
\hline 720.0222 & $1.93 E+06$ & $1.92 \mathrm{E}+06$ & $2.12 \mathrm{E}+06$ & $2.31 \mathrm{E}+06$ & $2.49 E+06$ & $2.58 \mathrm{E}+06$ & $2.84 \mathrm{E}+06$ & $2.70 E+06$ & -0.857 & 8.299 & {$\left[\mathrm{M}-\mathrm{Nic}+\mathrm{NaCaAc} c_{2}-2 \mathrm{H}\right]$} \\
\hline 724.0753 & $1.09 E+06$ & $1.27 \mathrm{E}+06$ & $1.38 \mathrm{E}+06$ & $1.74 \mathrm{E}+06$ & $2.08 \mathrm{E}+06$ & $2.24 \mathrm{E}+06$ & $2.46 \mathrm{E}+06$ & $2.28 \mathrm{E}+06$ & -0.929 & 10.013 & {$\left[\mathrm{M}+\mathrm{Na}_{2}(\mathrm{OH})-2 \mathrm{H}\right]^{-}$} \\
\hline 744.1055 & $1.86 \mathrm{E}+07$ & $1.74 \mathrm{E}+07$ & $1.64 \mathrm{E}+07$ & $1.39 \mathrm{E}+07$ & $1.09 \mathrm{E}+07$ & $7.81 \mathrm{E}+06$ & $2.15 E+06$ & $1.23 \mathrm{E}+05$ & 1.000 & 11.871 & {$[\mathrm{M}+\mathrm{NaAc}-\mathrm{H}]^{-}$} \\
\hline 745.1094 & $5.19 E+06$ & $5.03 E+06$ & $4.46 \mathrm{E}+06$ & $3.74 \mathrm{E}+06$ & $2.99 E+06$ & $1.97 \mathrm{E}+06$ & $3.63 E+05$ & $0.00 E+00$ & 1.000 & 9.871 & ${ }^{13} \mathrm{C}_{1^{-}}[\mathrm{M}+\mathrm{NaAC}-\mathrm{H}]^{-}$ \\
\hline 746.1118 & $1.09 \mathrm{E}+06$ & $1.13 \mathrm{E}+06$ & $7.56 \mathrm{E}+05$ & $5.54 \mathrm{E}+05$ & $4.36 \mathrm{E}+05$ & $1.78 \mathrm{E}+05$ & $0.00 E+00$ & $0.00 E+00$ & 0.909 & 7.871 & ${ }^{18} \mathrm{O}_{1}-[\mathrm{M}+\mathrm{NaAc}-\mathrm{H}]^{-}$ \\
\hline 760.0731 & $4.67 E+06$ & $5.16 \mathrm{E}+06$ & $6.09 E+06$ & $6.66 \mathrm{E}+06$ & $6.46 \mathrm{E}+06$ & $6.37 E+06$ & $5.13 E+06$ & $2.60 \mathrm{E}+06$ & 0.071 & 6.052 & {$[\mathrm{M}+\mathrm{CaAc}-2 \mathrm{H}]^{-}$} \\
\hline 761.0754 & $1.07 \mathrm{E}+06$ & $1.28 \mathrm{E}+06$ & $1.59 \mathrm{E}+06$ & $1.55 \mathrm{E}+06$ & $1.63 E+06$ & $1.65 E+06$ & $1.06 \mathrm{E}+06$ & $3.56 \mathrm{E}+05$ & 0.000 & 5.909 & ${ }^{13} \mathrm{C}_{1}-[\mathrm{M}+\mathrm{CaAc}-2 \mathrm{H}]^{-}$ \\
\hline 784.1068 & $1.22 \mathrm{E}+06$ & $1.21 \mathrm{E}+06$ & $8.11 E+05$ & $6.26 E+05$ & $2.37 E+05$ & $1.34 \mathrm{E}+05$ & $0.00 E+00$ & $0.00 E+00$ & 0.982 & 5.909 & {$\left[\mathrm{M}+\mathrm{Na}_{2}(\mathrm{OH}) \mathrm{Ac}-\mathrm{H}\right]^{-}$} \\
\hline 820.0956 & $4.63 E+06$ & $4.29 E+06$ & $3.74 \mathrm{E}+06$ & $3.28 \mathrm{E}+06$ & $2.17 E+06$ & $1.59 \mathrm{E}+06$ & $1.97 \mathrm{E}+05$ & $0.00 E+00$ & 1.000 & 3.945 & {$\left[\mathrm{M}+\mathrm{CaAc} \mathrm{C}_{2}-\mathrm{H}\right]^{-}$} \\
\hline 821.0914 & $1.10 \mathrm{E}+06$ & $1.16 \mathrm{E}+06$ & $6.32 \mathrm{E}+05$ & $5.88 \mathrm{E}+05$ & $3.22 E+05$ & $4.39 E+04$ & $0.00 E+00$ & $0.00 E+00$ & 0.909 & 1.945 & ${ }^{13} \mathrm{C}_{1}-\left[\mathrm{M}+\mathrm{CaAc} \mathrm{C}_{2}-\mathrm{H}\right]^{-}$ \\
\hline 902.0943 & $8.27 \mathrm{E}+05$ & $6.67 \mathrm{E}+05$ & $3.17 \mathrm{E}+05$ & $3.84 \mathrm{E}+05$ & $1.85 \mathrm{E}+05$ & $3.72 \mathrm{E}+04$ & $0.00 \mathrm{E}+00$ & $0.00 \mathrm{E}+00$ & 0.909 & 0.127 & {$\left[\mathrm{M}+\mathrm{NaCaAc}{ }_{3}-\mathrm{H}\right]^{-}$} \\
\hline
\end{tabular}

